\title{
Temporal Risk and the Nature of Induced Preferences*
}

\author{
MARK J. MACHINA \\ Department of Economics, University of California, \\ San Diego, La Jolla, California 92093 \\ Received December 8, 1982; revised April 18, 1983
}

\begin{abstract}
It has been known since the work of $\mathrm{H}$. Markowitz ("Portfolio Selection: Efficient Diversification of Investments," Yale Univ. Press, 1959) and J. Mossin (Amer. Econ. Rev. 59 (1969), 172-174) that even an individual whose underlying preferences satisfy the von Neumann-Morgenstern axioms will not choose over delayed (i.e., "temporal") risky prospects in a manner which can be modelled as expected utility maximizing. Since most economically important instances of risk taking (insurance, real investment, agriculture, career training) involve delayed as opposed to immediately resolved risk, the standard use of expected utility theory to model such decisions must be questioned. In this paper the technique of "generalized expected utility analysis" (M. J. Machina, Econometrica 50 (1982), 277-323) and the theory of support functions (R. T. Rockafellar, "Convex Analysis," Princeton Univ. Press, 1970) are applied to exactly model and hence determine the nature of preferences over temporal risky prospects. Journal of Economic Literature Classification Numbers: 022, 026.
\end{abstract}

\section{INTRODUCTION}

While it is well recognized that decisions of an intertemporal nature invariably contain some element of risk, it is only recently that economic theorists have given explicit recognition to the converse proposition, namely, that the resolution of uncertainty typically involves the passage of time. Decisions concerning insurance, agricultural production, portfolio composition, real investment, and career training, for example, all typically involve a significant interval between the time that the relevant decision must be made and the time that all uncertainty is completely resolved. Indeed, it is hard to think of any type of risk which does not involve the delayed resolution of uncertainty, other than the somewhat artificial (and in any event, completely avoidable) risks generated by some forms of gambling.

* I would like to thank David Baron, Frank Cowell. Vince Crawford, Frank Hahn, Beth Hayes, David Kreps, David Nachman, Larry Selden, Joel Sobel, Hugo Sonnenschein, Andrew Weiss, and an anonymous referee for helpful suggestions on this material. All errors, however. are my own. 
The fundamental sense in which choice over delayed risky prospects differs from the standard textbook case of choice over immediately resolved gambles is that in the delayed resolution case the individual is likely to have to make some other decision or decisions before the uncertainty of the chosen prospect is due to be resolved. ${ }^{1}$ In the literature (e.g., [15]), such a situation is said to involve "temporal risk," and we shall refer to these other decisions as intermediate or "auxiliary" decisions. The alternative case when the propects are either immediately resolved or else the individual faces no auxiliary decisions is referred to as that of "timeless risk."

To see how individuals will choose in situations of temporal risk, consider an individual who faces a set of alternative temporal prospects $\left\{\tilde{x}_{i}\right\}$ with respective cumulative distribution functions $\left\{F_{i}(\cdot)\right\}$, who must make some other auxiliary choice $\alpha$ out of a set $A$, and who seeks to maximize the expectation of a von Neumann-Morgenstern utility function $\phi(x, \alpha)$. In such a case, it is clear that the individual will rank each temporal prospect on the basis of how much expected utility it will yield given the optimal corresponding auxiliary decision, or in other words on the basis of the value of $Y\left(F_{i}\right) \equiv \int \phi\left(x, \alpha\left(F_{i}\right)\right) d F_{i}(x)$, where $\alpha\left(F_{i}\right)=\operatorname{argmax}_{\alpha \in A} \int \phi(x, \alpha) d F_{i}(x)$. The functional $Y(\cdot)$ is referred to as the individual's "induced preference functional" over temporal distributions, and the ranking it generates as the individual's "induced preferences." Note by way of contrast that if the prospects werc not temporal, i.c., if the choice of $\alpha$ could be postponed until after the uncertainty of the chosen prospect were resolved, then the individual would rank the prospects on the basis of the value of $W\left(F_{i}\right) \equiv$ $\int \phi(x, \alpha(x)) d F_{i}(x)$, where $\alpha(x)=\operatorname{argmax}_{\alpha \in A} \phi(x, \alpha)$. Needless to say, the functionals $W(\cdot)$ and $Y(\cdot)$ will typically not be ordinally equivalent.

While it is straightforward to show that induced preferences over temporal prospects will be complete, transitive, and continuous, the fundamental result in the theory of temporal risk is the observation by Markowitz $[42$, Chap. 11], Mossin [47], and Spence and Zeckhauser [68] that such preferences will typically not satisfy the final, and key, axiom of expected utility theory, namely, the so-called "independence axiom" (see, e.g., [62]). In other words, while the preference functional $W(F) \equiv \int \phi(x, \alpha(x)) d F(x)$ remains a linear functional of $F(\cdot)$ ("linear in the probabilities") and hence can be represented as the expectation of the von Neumann-Morgenstern utility function $\xi(x) \equiv \phi(x, \alpha(x))$, there will in general exist no von Neumann-Morgenstern utility function capable of summarizing the ranking generated by the (nonlinear) functional $Y(F) \equiv \int \phi(x, \alpha(F)) d F(x)$. In particular, rather than being "linear," induced preferences over temporal

\footnotetext{
${ }^{1}$ If nothing else, the individual will presumably be making consumption-savings decisions in the meantime.
} 
prospects are in fact "quasiconvex in the probabilities," so that an individual who is indifferent between the temporal prospects with respective distributions $F_{a}(\cdot)$ and $F_{b}(\cdot)$ will weakly (and in general, strictly) prefer them to the temporal prospect with distribution $F_{c}(\cdot)=\frac{1}{2} F_{a}(\cdot)+\frac{1}{2} F_{b}(\cdot)$.

Needless to say, the implications of this fact for the modelling of decisions under uncertainty are quite important. If temporal as opposed to timeless prospects are indeed, as Mossin argues, "the rule rather than the exccption" $[47$, p. 174], then the fact that preferences over temporal prospects systematically depart from linearity implies that the use of atemporal expected utility theory to model such decisions would at best be a hopeful approximation and at worst lead to systematically incorrect results and predictions (see also the remarks of Kreps and Porteus [31, p. 82] on this point).

One way to account for this problem (and to retain the use of expected utility theory) is to explicitly incorporate the exact nature of the auxiliary decisions each individual faces in such situations, and then to derive and do comparative statics on the jointly optimal risky propect and auxiliary decision(s). However, the fact that such auxiliary decisions will typically be multi-dimensional, differ across individuals, and often be of little interest to the researcher has led Kreps and Porteus to conclude that "the obvious difficulty with this approach is that such complete models may become overburdened with detail and analytically intractable" $[31$, p. 83$]$. Since it will usually be impractical to leave all auxiliary decisions in such models, and theoretically incorrect to use the expected utility approach when they are maximized out, they conclude that "we are forced to develop preference structures which can be used to model induced preferences directly" $[31$, p. 83]. This procedure of maximizing out one component of a joint decision problem in order to more directly study preferences over another is a standard one in economics - it is invoked in the construction of trade indifference curves (e.g., [11, Chapter 6]), the use of the Hicks composite commodity theorem in order to do indifference curve analysis in two dimensions [61, Chapter 4 ], the derivation of preferences over current transactions in temporary general equilibrium theory $[6$, Section $6.3 ; 21$, p. 540], and, indeed, even in the use of indirect utility functions (i.e., utility of wealth functions) in expected utility theory. General discussions of the nature and advantages of this "two-stage" or "induced preferences" approach to economic analysis are offered in Cox [13], Liviatan [34], and Milne [44].

Kreps and Porteus [31, Propositions 5 and 6] have shown that under certain conditions on the underlying utility function $\phi(x, \alpha)$, the individual's induced preferences may be represented by a linear and hence expected utility preference ranking. However, they note that these conditions are "quite stringent" [31, p. 101] (essentially, $\phi(x, \alpha)$ must take the form $f(\alpha)+$ $g(a) h(x))$ and are unlikely to be generally satisfied. In light of this, they 
proceed to develop conditions under which induced preferences may be reasonably approximated by expected utility preferences. While the condition they propose $(\phi(x, \alpha)$ increasing in $x)$ is much less restrictive, they note that there is no-well-defined sense in which theirs is the "best" expected utility approximation of the individual's actual induced preferences, nor is it clear how (or whether) "closeness" of such an approximation of preferences implies "closeness" of the corresponding behavior (e.g., insurance or asset demand) functions.

The purpose of this paper is to show that, provided conditions are sufficiently "smooth," it is possible to use "expected utility" analysis to exactly model induced preferences over temporal prospects, whether or not such preferences are linear in the probabilities. The analytical techniques used will be those developed in [38], which demonstrated how expected utility analysis may be applied to nonlinear preferences in precisely the same manner in which calculus uses the techniques of linear algebra to provide (exact, global) results relating the behavior of a function and its derivatives (i.e., its local linear approximations). Results from [38], along with generalizations of standard results in the theory of support functions (e.g., Rockafellar [55|), are used to derive general properties of induced preferences, and in particular whether (and when) properties of underlying preferences will carry over to induced preferences. By identifying which properties of underlying preferences are likely to carry over to induced preferences, which are not, and which new properties (such as quasiconvexity) are introduced, we are able to impose more realistic assumptions on the preferences, and hence behavior, of individuals in situations of temporal risk.

The following section offers a brief summary of results from [38] (see also the exposition in [41]). The application of this approach to the analysis of induced preferences over temporal prospects, including how properties of underlying preferences (such as risk aversion, declining risk aversion, etc.) carry over, is undertaken in Section 3. Section 4 applies these results to some common examples of temporal risk: delayed income prospects ("income risk"), delayed returns to investment ("capital risk"), and multiple risky decisions. The paper concludes (Section 5) with a result linking the analysis with the experimental literature on choice under uncertainty.

\section{The Analysis of General Nonlinear Preference functionals}

Taking as our choice set the set $D[0, M]$ of all cumulative distribution functions $F(\cdot)$ over the wealth interval $[0, M]$, we assume that the individual's preferences over $D[0, M]$ are complete, transitive, and representable by a real-valued preference functional $V(\cdot)$ which is continuous and 
"smooth" in the sense of being (once) Frechet differentiable. In [38] this was shown to imply that at each $F_{0}(\cdot)$ in $D[0, M]$ there will exist a "local utility function" $U\left(\cdot ; F_{0}\right)$ over $[0, M]$ such that for any other $F(\cdot)$ in $D[0, M]$,

$$
V(F)-V\left(F_{0}\right)=\int U\left(x ; F_{0}\right)\left[d F(x)-d F_{0}(x)\right]+o\left(\left\|F-F_{0}\right\|\right),
$$

where $o(\cdot)$ is a function of higher order than its argument, and $\|\cdot\|$ is the $L^{1}$ norm $\int\left|F(x)-F_{0}(x)\right| d x$. Thus, just as in standard calculus, the difference $V(F)-V\left(F_{0}\right)$ is seen to consist of a first order (i.e., linear) term in $F(\cdot)-F_{0}(\cdot)$ plus a higher-order term, where in this case the linear term can be represented as the difference in the expectation of $U\left(\cdot ; F_{0}\right)$ with respect to the distributions $F(\cdot)$ and $F_{0}(\cdot)$, so that in ranking alternative differential shifts from $F_{0}(\cdot)$ the individual will act precisely as would an expected utility maximizer with von Neumann-Morgenstern utility function $U\left(\cdot ; F_{0}\right)$. Thus, for example, the individual will be averse to all differential mean preserving increases in risk about $F_{0}(\cdot)$ (see [58]) if and only if $U\left(x ; F_{0}\right)$ is concave in $x$.

We may extend this approach to the global analysis of nonlinear preference functionals by means of another analogy with standard calculus, namely, the use of path integrals and the Fundamental Theorem of Integral Calculus. Say, for example, that $F(\cdot)$ differs from $F_{0}(\cdot)$ by a "large" mean preserving increase in risk. Defining the path $\{F(\cdot ; \beta) \mid \beta \in[0,1]\}$ from $F_{0}(\cdot)$ to $F(\cdot)$ by $F(\cdot ; \beta) \equiv \beta F(\cdot)+(1-\beta) F_{0}(\cdot)$, we see that each differential increase in $\beta$ will induce a differential mean preserving increase in risk. Since $V(F)-V\left(F_{0}\right)$ will equal the integral of $d(V(\cdot ; \beta)) / d \beta$ as $\beta$ runs from 0 to 1 , and since each of these differential shifts will be preferred or not preferred depending upon whether it raises or lowers the expectation of the relevant local utility function $U(\cdot ; F(\cdot ; \beta))$ at the point on the path, it follows, for example, that if the local utility functions are everywhere concave in $x$ then this global mean preserving increase in risk will not be preferred by $V(\cdot)$. In [38] this approach was used to demonstrate that the "expected utility" condition of concavity of (all of) the local utility functions was a necessary and sufficient condition for global risk aversion in this sense, even though the individual is not necessarily an expected utility maximizer (i.e., even though $V(\cdot)$ may be nonlinear). Similarly, the condition that all the local utility functions be nondecreasing in $x$ was shown to be equivalent to the property of (global) "monotonicity," i.e., preference for first-order stochastically dominating distributions.

In order to extend the expected utility characterization of comparative risk aversion, we adopt the following generalization of the Diamond-Stiglitz notion of a "mean utility preserving increase in risk" [14, pp. 341-42]:

Definitions [38, p. 281]. If $F(\cdot)$ and $F^{*}(\cdot)$ are in $D[0, M]$, then $F^{*}(\cdot)$ 
is said to differ from $F(\cdot)$ by a simple compensated spread if the individual is indifferent between $F^{*}(\cdot)$ and $F(\cdot)$ and if $F^{*}(x) \geqslant(\leqslant) F(x)$ as $x \leqslant(\geqslant) x^{*}$ for some $x^{*} \in[0, M], F^{*}(\cdot)$ is said to differ from $F(\cdot)$ by a compensated increase in risk if it differs from $F(\cdot)$ by a sequence of simple compensated spreads.

THEOREM [38, Theorem 4]. The following conditions on a pair of Fréchet differentiable functions $V(\cdot)$ and $V^{*}(\cdot)$ over $D[0, M]$ with respective local utility functions $U(\cdot ; F)$ and $U^{*}(\cdot ; F)$ are equivalent:

(i) $U(x ; F)$ is at least as concave a function of $x$ as is $U^{*}(x ; F)$, so that if these functions are twice differentiable in $x$, then $-U_{11}(x ; F) / U_{1}(x ; F) \geqslant-U_{11}^{*}(x ; F) / U_{1}^{*}(x ; F)$ for all $x$ in $[0, M]$ and $F(\cdot)$ in $D[0, M]$, where subscripts denote successive partial derivatives with respect to $x$;

(ii) If $F^{*}(\cdot)$ differs from $F(\cdot)$ by a compensated increase in risk from the point of view of $V^{*}(\cdot)$, then $V\left(F^{*}\right) \leqslant V(F)$; and

(iii) If $F(\cdot)$ is indifferent between a $p:(1-p)$ chance of $F(\cdot)$ or $F^{*}(\cdot)$ and $a p:(1-p)$ chance of the sure amount $c$ or the distribution $F^{*}(\cdot)$, and $V^{*}(\cdot)$ is indifferent between a $p:(1-p)$ chance of $F(\cdot)$ or $F^{*}(\cdot)$ and a $p:(1-p)$ chance of the sure amount $c^{*}$ or $F^{*}(\cdot)$, then $c \leqslant c^{*}$ (i.e., the "conditional certainty equivalents" $[38$, p. 288] of $V(\cdot)$ are never greater than those of $\left.V^{*}(\cdot)\right)$.

If both individuals are "diversifiers" [38, p. 299] and have differentiable local utility functions, then the above conditions are also equivalent to:

(iv) For any $F^{* *}(\cdot) \in D[0, M], p \in(0,1], r>0$, and nonnegative random variable $\tilde{z}$ with mean greater than $r$, if $\gamma$ and $\gamma^{*}$ yield the most preferred distributions of the form $(1-p) F^{* *}+p F_{(1-\gamma) r+\gamma \tilde{z}}$ for $V(\cdot)$ and $V^{*}(\cdot)$, respectively, ${ }^{2}$ then $\gamma \leqslant \gamma^{*}$ (i.e., the "conditional demands for risky assets" [38, p. 298] of $V(\cdot)$ are never greater than those of $\left.V^{*}(\cdot)\right)$.

In our later discussion of induced preferences it will be crucial that we understand the precise sense in which the above theorem generalizes the Arrow/Pratt/Diamond and Stiglitz $[5,52,14]$ characterization of comparative risk aversion to the general nonexpected utility case. In particular, it is important to note that the fact that one individual always assigns lower certainty equivalents than another is no longer a sufficient condition for the first individual to be more risk averse in any of the other senses. To see why this should be so, consider the following set of conditions on a pair of individuals (conditions (i) and (ii) below are taken from the above theorem):

\footnotetext{
${ }^{2} F_{(1-\gamma) r+\gamma \bar{z}}(\cdot)$ is defined as the cumulative distribution function of the random variable $(1-\gamma) r+\gamma \tilde{z}$.
} 
(E) both individuals are expected utility maximizers;

(i) the first individual has more concave local utility functions than the second;

(ii) compensated increases in risk for the second individual make the first individual worse off;

(iii*) the first individual always assigns lower certainty equivalents than the second; and

(iv*) the first individual always demands less of the risky asset than the second.

Assuming for simplicity that both individuals are diversifiers, there are two ways to represent the Arrow/Pratt/Diamond and Stiglitz theorem, i.e., as the conditional equivalence of (i), (ii), (iii*), and (iv*) given $E$, or as the unconditional equivalence of the four joint conditions " $E$ and (i)," " $E$ and (ii)," "E and (iii*)," and "E and (iv*)." Generalizing the first representation to the nonexpected utility case would mean dropping the phrase "given E," leaving the "equivalence" of (i), (ii), (ii*), and (iv*). However, since (i) and (ii) refer to properties of preferences over every small neighbourhood in $D[0, M]$ while (iii*) and (iv*) do not, it turns out that in the absence of any additional restrictions such as linearity neither the certainty equivalent characterization (iii*) nor the asset demand characterization (iv*) is strong enough to imply condition (i) or (ii), or more important still, even each other. Rather, the correct approach is to generalize the unconditional version of the theorem by finding those natural generalizations of the conditions " $E$ and (iii*)" and "E and (iv*)" which refer to properties of preferences in every locality of $D[0, M]$, and it may be shown that conditions (iii) and (iv) of the above theorem are precisely those generalizations (see [38, Section 3.3 for further discussion). Accordingly, we shall say that one individual is "everywhere more risk averse" than another when they satisfy the conditions of the theorem, and in subsequent sections shall return to the fact that it is the "conditional certainty equivalent" property (iii) rather than the weaker property (iii*) which is the appropriate "certainty equivalent" characterization of comparative risk aversion in the general (i.e., nonexpected utility) case.

\section{The Analysis of Induced Preferences over Temporal Prospects}

In this section we analyze the nature of induced preferences over temporal prospects by studying properties of the induced preference functional $Y(F) \equiv$ $\max _{\alpha \in A} \int \phi(x, \alpha) d F(x) \equiv \int \phi(x, \alpha(F)) d F(x)$. 
The first useful property of $Y(\cdot)$ is its specific functional structure. Defining the "conditional preference functional" $Y(\cdot ; \alpha): D[0, M] \rightarrow R^{1}$ by $Y(F ; \alpha) \equiv \int \phi(x, \alpha) d F(x)$, we see that as the pointwise extremum of the set of linear functionals $\{Y(\cdot, \alpha) \mid \alpha \in A\}$, the functional $Y(F)\left(\equiv \max _{\alpha \in A} Y(F ; \alpha)\right)$ is an example of what is termed a "support function" in convex analysis (see, e.g., Rockafellar [55, Chapter 13]). Other examples of support functions in economics include the profit function of the firm and the (utility constrained) expenditure function of the individual, and in Section 3(a)-3(c) we exploit this analogy to obtain several results which correspond to the elegant and powerful results in the modern "dual approach" to microeconomics (e.g., Varian [74]).

Perhaps not surprisingly, the most important of these results will be that, under very weak conditions on the underlying utility function $\phi(\cdot, \cdot)$ and auxiliary choice set $A, Y(\cdot)$ will be Fréchet differentiable. This fact will allow us to apply the technique of "generalized expected utility analysis" summarized in Section 2, allowing for the derivation of further results in the theory of induced preferences (Sections 3(d) - 3(i)).

Throughout this paper we shall assume that the set $A$ is nonempty and compact, that $\phi(x, \alpha)$ is continuous and increasing in $x$, and that the conditional preference functional $Y(\cdot ; \alpha)$ varies continuously in $\alpha$ with respect to the standard operator norm $\|Y(\cdot ; \alpha)\| \equiv \sup _{F, F^{*} \in D[0, M]}\left|Y\left(F^{*}-F ; \alpha\right)\right| /$ $\left\|F^{*}-F\right\| .{ }^{3}$ Wc also usc the symbol $G_{c}(\cdot)$ to denote the distribution which assigns unit probability to the point $c$, and $F_{+c}(\cdot)$ (resp. $\left.F_{\times c}(\cdot)\right)$ as the distribution obtained by translating the distribution $F(\cdot) c$ units to the right (resp. scaling up the distribution $F(\cdot)$ by a factor of $c$ about the origin), so that $F_{+c}(x) \equiv F(x-c)$ and $F_{x c}(x) \equiv F(x / c)$.

\section{(a) Continuity and Convexity}

Under the above assumptions the induced preference functional $Y(\cdot)$ is a special case of that studied by Kreps and Porteus [31], and the following result is obtained as a special case of their Lemma 1 and Proposition 3:

THEOREM 1. The induced preference functional $Y(\cdot)$ is continuous and convex over $D[0, M]$ in the topology of weak convergence (see, e.g., [7]), and the auxiliary choice correspondence $\alpha(F) \equiv \operatorname{argmax}_{\alpha \in A} \int \phi(x, \alpha) d F(x)$ is upper semicontinuous.

Not surprisingly, the proofs of continuity and convexity here parallel almost exactly the corresponding proofs for profit and expenditure functions.

${ }^{3}$ This will be true, for example, if $d \phi(x, \alpha) / d \alpha$ exists and is continuous in $(x, \alpha)$. Note also that while we assume the set $A$ to be independent of the chosen $F(\cdot)$, many of our results will extend to the more general case when $A$ depends upon $F(\cdot)$. 


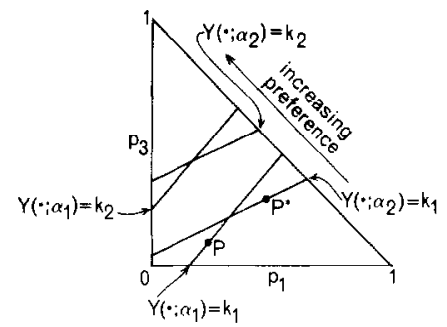

FIG. 1. Construction of indifference curves of induced preferences from indifference curves of underlying preferences.

It will be useful to illustrate this and certain subsequent results in the special case of preferences over the set $D\left\{x_{1}, x_{2}, x_{3}\right\}$ of all probability distributions over the outcomes $x_{1}<x_{2}<x_{3}$ in $[0, M]$, which may be represented by the points in the unit triangle in the $\left(p_{1}, p_{3}\right)$ plane as in Fig. 1, where $p_{2}=\operatorname{prob}\left(x_{2}\right)=1-p_{1}-p_{3}$ (see also Markowitz [42, p. 251]). The "linear" preferences of an expected utility maximizer will generate indifference curves here which are straight, parallel, upward sloping, and more preferred as we move northwest, as illustrated by the two sets of parallel linear indifference curves in Fig. 1, which correspond to the underlying utility functions $\phi\left(\cdot, \alpha_{1}\right)$ and $\phi\left(\cdot, \alpha_{2}\right)$ (i.e., the conditional preference functionals $Y\left(\cdot ; \alpha_{1}\right)$ and $\left.Y\left(\cdot ; \alpha_{2}\right)\right)$, respectively. At "point" (i.e., distribution) $P$ the individual will be led to make the auxiliary choice $\alpha_{1}$ (which yields expected utility $k_{1}$ ) over $\alpha_{2}$ (which yields a lower expected utility), and at $P^{*}$ will be led to choose $\alpha_{2}$, which implies that when the auxiliary choice set is $\left\{\alpha_{1}, \alpha_{2}\right\}$ the $Y(\cdot)=k_{1}$ indifference curve will be the lower envelope of the $Y\left(\cdot, \alpha_{1}\right)=k_{1}$ and $Y\left(\cdot ; \alpha_{2}\right)=k_{1}$ indifference curves. This illustrates why $Y(\cdot)$ must be at least quasiconvex (the case where $A$ is a continuum is shown in Fig. 2). An important implication of this result is that generalizations of expected utility theory which do not admit of the case of strictly quasiconvex preferences (e.g., Chew [12]) will be similarly inapplicable in situations of temporal risk.

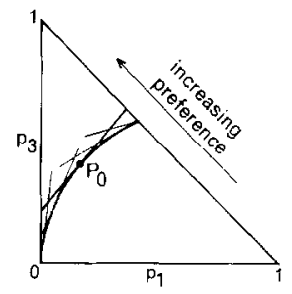

FIG. 2. Construction of indifference curves of induced preferences when $A$ is a continuum. 
The cardinal property of convexity of $Y(\cdot)$ turns out to be relevant in determining choice over immediately resolved lotteries over temporal prospects, where it can be shown that the individual will seek to maximize the expectation of $Y(\cdot)$. Convexity here implies that the individual will prefer an immediate $p:(1-p)$ lottery over the temporal distributions $F_{a}(\cdot)$ and $F_{b}(\cdot)$ to the temporal distribution $p F_{a}(\cdot)+(1-p) F_{b}(\cdot)$ (for more on such "mixed timeless/temporal lotteries," see Kreps and Porteus [31] and Epstein [18]).

\section{(b) Characterization and Recoverability}

The following theorem demonstrates that the above properties of continuity and convexity form a complete characterization of induced preferences, in that any continuous convex functional $V(\cdot)$ will be the induced preference functional for some set $\{U(\cdot)\}$ of underlying von Neumann-Morgenstern utility functions:

THEorem 2. If $V: D[0, M] \rightarrow R^{1}$ is continuous and convex, then $V(F) \equiv \max _{U(\cdot) \in Z^{*}} \int U(x) d F(x)$, where $\quad Z^{*}=\{$ continuous $\quad U:[0, M] \rightarrow$ $R^{1} \mid \int U(x) d F(x) \leqslant V(F)$ for all $\left.F(\cdot) \in D[0, M]\right\}$.

\section{(Proof in Appendix.)}

Besides a characterization, Theorem 2 also offers a way of "recovering" a set of underlying utility functions $\left(Z^{*}\right)$ from an arbitrary continuous convex functional which serves to generate that functional as its induced preference functional. Since the method of proof is formally analogous to the "recovery" of the technology set from a profit function [74], it is natural to ask the analogous next question, namely, if $Y(\cdot)$ is the induced preference functional generated by the set $Z=\{\phi(\cdot, \alpha) \mid \alpha \in A\}$ of underlying utility functions, how will the "recovered" set $Z^{*}$ differ from $Z$ ? To answer this, again by analogy with profit functions, we define $C$ as the set of all continuous functions from $[0, M]$ to $R^{1}$ endowed with the topology of uniform convergence and adopt:

Definitions. A subset $B$ of $C$ is comprehensive if $U(\cdot) \in B, U^{*}(\cdot) \in C$, and $U^{*}(x) \leqslant U(x)$ for all $x \in[0, M]$ implies $U^{*}(\cdot) \in B$. The convex comprehensive closure of a set $B$ in $C$ is the smallest closed comprehensive subset of $C$ which contains $B$ (i.e., the intersection of all closed comprehensive subsets of $C$ which contain $B$ ).

THEOREM 3. If $Y(F) \equiv \max _{\alpha \in A} \int \phi(x, \alpha) d F(x) \equiv \max _{U(\cdot) \in Z} \int U(x)$ $d F(x)$, where $Z=\{\phi(\cdot, \alpha) \mid \alpha \in A\}$, then the set $Z^{*}=\{$ continuous $U:[0, M] \rightarrow R^{1} \mid \int U(x) d F(x) \leqslant Y(F)$ for all $\left.F(\cdot) \in D[0, M]\right\}$ is the convex comprehensive closure of $Z$. 


\section{(Proof in Appendix.)}

The fact that $Z^{*}$ contains the convex hull of $Z$ might seem to imply that it has "recovered" substantially more than the individual's actual underlying preferences, since for any two functions $\phi\left(\cdot, \alpha_{1}\right)$ and $\phi\left(\cdot, \alpha_{2}\right)$ in $Z, Z^{*}$ will contain the function $\frac{1}{2} \phi\left(\cdot, \alpha_{1}\right)+\frac{1}{2} \phi\left(\cdot, \alpha_{2}\right)$, whether or not this function is in $Z$ (recall that if the original technology set is not convex the "recovered" technology set will be strictly larger). However, unlike the case of profit functions, this does not really represent an "expansion" of the individual's preferences or opportunities, since an individual with utility functions $\phi\left(\cdot, \alpha_{1}\right)$ and $\phi\left(\cdot, \alpha_{2}\right)$ could always do at least as well with one of these functions as with the mixture $\frac{1}{2} \phi\left(\cdot, \alpha_{1}\right)+\frac{1}{2} \phi\left(\cdot, \alpha_{2}\right)$, and in any event could always exactly attain the expected utility given by this latter function by taking an immediately resolved 50:50 randomization over $\alpha_{1}$ and $\alpha_{2}$. Similarly, since the individual is attempting to maximize expected utility, the fact that $Z^{*}$ will recover "dominated" utility functions (i.e., is comprehensive) also represents no real expansion of the individual's preferences or opportunities.

Since Theorem 3 required knowledge of the cardinal functional $Y(\cdot)$, it is natural to ask whether underlying preferences can be recovered solely from the individual's ordinal preferences over temporal prospects. Since the sets \{continuous $U:[0, M] \rightarrow R^{1} \mid \int U(x) d F(x) \leqslant Y(F)$ for all $\left.F(\cdot)\right\}$ and $\{$ continuous $U:[0, M] \rightarrow R^{1} \mid \int U(x) d F(x) \leqslant \delta(Y(F))$ for all $\left.F(\cdot)\right\}$ are not equal for arbitrary monotonic $\delta(\cdot)$, it follows that ordinal preferences alone will not suffice. Theorem 4, however, shows that it is possible to recover $Y(\cdot)$, and hence underlying preferences, from knowledge of the ordinal ranking over the union of the set of temporal prospects and the set of immediately resolved "basic reference lotteries" (i.e., lotteries over the two outcomes 0 and $M$ ).

THEOREM 4. Under the assumptions of the beginning of Section 3, for each $F(\cdot)$ in $D[0, M]$ there exists a unique probability $p$ such that the individual is indifferent between the temporal distribution $F(\cdot)$ and an immediately resolved $p:(1-p)$ chance of $M$ or 0 (see [31, Lemma 4(b)]). In addition, the map $F(\cdot) \rightarrow p$ is cardinally equivalent to the induced preference functional $Y(\cdot)$.

\section{(Proof in Appendix.)}

\section{(c) Differentiability}

Two of the "nice" properties of support functions are the relatively weak conditions needed for their differentiability and the "envelope" results concerning their derivatives. Thus, for example, for a profit function $\pi(P) \equiv$ $\max _{X \text { feasible }} P \cdot X$ to be differentiable at a price vector $P_{0}$, it is not necessary for the production function or the technology frontier to be differentiable, 
merely that the profit maximizing net output vector $X(P)$ be both unique and upper semicontinuous at $P_{0}$. Defining the "conditional (on $X$ ) profit function" $\pi(\cdot ; X)$ by $\pi(P ; X) \equiv P \cdot X$, we have that in such a case the derivative of $\pi(\cdot)$ at $P_{0}$ will be precisely the conditional profit function $\pi\left(\cdot ; X\left(P_{0}\right)\right)$ (i.e., the partial derivatives of $\pi(\cdot)$ are the net supplies). Similarly, in order for $Y(F) \equiv \int \phi(x, \alpha(F)) d F(x)$ to be Fréchet differentiable at $F_{0}(\cdot)$ it is neither necessary for $\phi(x, \alpha)$ to be differentiable in $\alpha$ nor for $\alpha(F)$ to be differentiable in $F(\cdot)$, but merely that the optimal auxiliary choice $\alpha(F)$ be both unique and upper semicontinuous at $F(\cdot)$ (recall that upper semicontinuity follows from Theorem 1$)$. In this case, it similarly follows that the derivative of $Y(\cdot)$ at $F_{0}(\cdot)$ is the conditional preference functional $Y\left(\cdot ; \alpha\left(F_{0}\right)\right)$, or in other words, that the local utility function of $Y(\cdot)$ at $F_{0}(\cdot)$ is precisely $\phi\left(\cdot, \alpha\left(F_{0}\right)\right)$.

THEOREM 5. If $\alpha\left(F_{0}\right)$ is unique and $\alpha(\cdot)$ is upper semicontinuous at $F_{0}(\cdot)$, then $Y(F) \equiv \int \phi(x, \alpha(F)) d F(x)$ will be Fréchet differentiable at $F_{0}(\cdot)$ with Fréchet derivative $Y\left(\cdot ; \alpha\left(F_{0}\right)\right)$, and hence with local utility function $\phi\left(\cdot, \alpha\left(F_{0}\right)\right)$.

\section{(Proof in Appendix.)}

To see this result graphically, note that when the linear indifference curves generating the lower envelope vary continuously as in Fig. 2, the resulting envelope will be "smooth," and also that the linear approximation to preferences at a distribution $P_{0}$ will be precisely that linear preference field generated by the utility function $\phi(\cdot, \alpha)$ at the optimal choice $\alpha\left(P_{0}\right)$ (i.e., the heavy straight line in Fig. 2). ${ }^{4}$

It is clear that this result offers a very powerful tool for the analysis of induced preferences. One way to phrase the problem posed by the prevalence of temporal as opposed to instantaneous risk is that while the nature of choice will be determined most directly by the induced preference ranking, all of our knowledge and intuition concerning preferences concerns the underlying utility function $\phi(x, \alpha)$. However, Theorem 5 provides a fundamental link between the two: it tells us that the local utility functions of the induced preference functional, with which we can analyze its nature (as in Section 2), are precisely the corresponding underlying utility functions, about which we know a lot. In light of the usefulness of this approach, we shall assume for the remainder of this paper that $\alpha(F)$ is everywhere unique, and hence by Theorem 1, everywhere continuous in $F(\cdot)$.

\footnotetext{
${ }^{4}$ Figure 2 also illustrates the Diamond and Stiglitz $[14$, p. 343$]$ result that compensated increases in the riskiness of $F(\cdot)$ will change $\alpha(F)$ so as to lower the risk aversion (i.e.. concavity) of $\phi(\cdot, \alpha)$ : we see from the figure that compensated increases in risk, which correspond to northeast movements along indifference curves, lead to chosen utility functions with less steeply sloped indifference curves, i.e., which are less concave (see |41, p. 267|).
} 
(d) Monotonicity, Risk Aversion, and Third Order Stochastic Dominance Preference

Three immediate implications of the above result are:

THEOREM 6. Under the above assumptions:

(i) $Y\left(F^{*}\right) \geqslant Y(F)$ whenever $F^{*}(\cdot)$ first order stochastically dominates $F(\cdot)$ if and only if $\phi(x, \alpha)$ is nondecreasing in $x$ for all $\alpha \in\{\alpha(F) \mid F(\cdot) \in$ $D[0, M]\}$;

(ii) $Y\left(F^{*}\right) \leqslant Y(F)$ whenever $F^{*}(\cdot)$ differs from $F(\cdot)$ by a mean preserving increase in risk if and only if $\phi(x, \alpha)$ is concave in $x$ for all $\alpha \in\{\alpha(F) \mid F(\cdot) \in D[0, M]\}$; and

(iii) If $\phi(x, \alpha)$ is thrice differentiable in $x$ for all $\alpha$, then $Y\left(F^{*}\right) \geqslant Y(F)$ whenever $F^{*}(\cdot)$ third order stochastically dominates $F(\cdot)$ [76] if and only if $\phi_{111}(x, \alpha) \geqslant 0$ for all $x$ and $\alpha \in\{\alpha(F) \mid F(\cdot) \in D[0, M]\}$.

(Proof in Appendix.)

Part (ii) of the theorem, for example, may be illustrated graphically in terms of the above "unit triangle diagram." Since it can be shown that one point (i.e., distribution) will differ from another by a mean preserving spread if and only if it lies to the northeast of that point along a line with slope $\left(x_{2}-x_{1}\right) /\left(x_{3}-x_{2}\right)[41]$, it follows that a set of indifference curves (linear or otherwise) will be risk averse (i.e., made worse off by such spreads) if and only if the curves are everywhere steeper than this slope. Figure 3 shows that if each of the underlying utility functions is risk averse, the envelopes they generate will be similarly steeper than this slope, so that induced preferences will also be risk averse (the dotted lines in the figure are lines with slope $\left.\left(x_{2}-x_{1}\right) /\left(x_{3}-x_{2}\right)\right)$.

While straightforward to establish, these results are important ones, for they imply that the above three economically important and widely assumed properties of underlying preferences will indeed carry over to induced preferences. Thus, for example, there is good reason to believe that those of our behavioral results and predictions which depend upon the assumption of

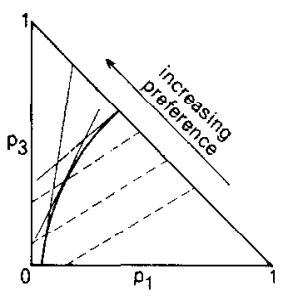

FIG. 3. Risk averse underlying preferences generate risk averse induced preferences. 
risk aversion will be robust to the typically temporal nature of decisions under uncertainty.

\section{(e) Comparative Risk Aversion}

Because an important source of comparative statics results under uncertainty concern the effect of "greater risk aversion" on behavior, it is natural to ask whether more risk averse underlying preferences lead to more risk averse preferences over temporal prospects. A partial answer to this question is given by the following generalization of a result due to Caperaa and Eeckhoudt $[10]$ :

THEOREM 7. Let $\rho(\cdot)$ be an increasing concave function and define $Y^{*}(F) \equiv \max _{\alpha \in A} \int \phi^{*}(x, \alpha) d F(x) \equiv \int \phi^{*}\left(x, \alpha^{*}(F)\right) d F(x)$, where $\phi^{*}(x, \alpha) \equiv$ $\rho(\phi(x, \alpha))$ and $\alpha^{*}(F) \equiv \operatorname{argmax}_{\alpha \in A} \int \phi^{*}(x, \alpha) d F(x)$. Then, if $c$ and $c^{*}$ solve $Y\left(G_{c}\right)=Y(F)$ and $Y^{*}\left(G_{c^{*}}\right)=Y^{*}(F)$, then $c \geqslant c^{*}$.

(Proof in Appendix.)

Thus, the more risk averse the underlying preferences, the lower the certainty equivalent (or equivalently, the higher the risk premium) assigned to any temporal prospect.

However, since induced preferences are typically not expected utility maximizing, it follows from the discussion in Section 2 that the above result is not enough to guarantee that $Y^{*}(\cdot)$ will be more risk averse in any of the other standard senses, i.e., that $Y^{*}(\cdot)$ will be "everywhere more risk averse than $Y(\cdot)$ " (see Section 2). To see why, recall that this condition requires that the local utility functions $\left\{\phi^{*}\left(\cdot, \alpha^{*}(F)\right)\right\}$ of $Y^{*}(\cdot)$ be at least as concave as the corresponding local utility functions $\{\phi(\cdot, \alpha(F))\}$ of $Y(\cdot)$. However, while $\phi^{*}(\cdot, \alpha)=\rho(\phi(\cdot, \alpha))$ will be at least as concave as $\phi(\cdot, \alpha)$ for given $\alpha$, it is quite possible for the change in $\alpha$ from $\alpha(F)$ to $\alpha^{*}(F)$ to more than offset this effect.

In the case of a univariate auxiliary choice set, the following theorem gives conditions under which concavifications of the underlying utility function will always lead to everywhere more risk averse preferences over temporal prospects.

THEOREM 8. If $A$ is an interval in $R^{1}$ and $\phi(\cdot, \cdot)$ is thrice continuously differentiable with $\phi_{22}(\cdot, \cdot)<0$, then the following conditions are equivalent:

(i) $-\phi_{11}\left(x, \alpha\left(G_{c}\right)\right) / \phi_{1}\left(x, \alpha\left(G_{c}\right)\right)$ is nonincreasing in $c$ over $[0, M]$;

(ii) $-\phi_{11}(x, \alpha) / \phi_{1}(x, \alpha)$ and $\alpha\left(G_{c}\right)$ are weakly monotonic in opposite directions in $\alpha$ and $c$, respectively, for all $\alpha \in\{\alpha(F) \mid F(\cdot) \in D[0, M)\}$ and $x$, $c \in[0, M]$; and

(iii) If $Y^{*}(F) \equiv \max _{\alpha \in A} \int \rho(\phi(x, \alpha)) d F(x)$, then $Y^{*}(\cdot)$ will be 
everywhere at least as risk averse as $Y(\cdot)$ whenever $\rho^{\prime}(\cdot)>0$ and $\rho^{\prime \prime}(\cdot) \leqslant 0$, and $Y(\cdot)$ will be everywhere at least as risk averse as $Y^{*}(\cdot)$ whenever $\rho^{\prime}(\cdot)>0$ and $\rho^{\prime \prime}(\cdot) \geqslant 0$.

\section{(Proof in Appendix.)}

Note that since $\phi\left(\cdot, \alpha\left(G_{c}\right)\right)$ is the local utility function of $Y(\cdot)$ at $G_{c}(\cdot)$, condition (i) may be verified from knowledge of the functional $Y(\cdot)$ alone. However, it is condition (ii) which is in fact the most applicable: not only is monotonicity of $\alpha\left(G_{c}\right)$ in $c$ an easily interpretable behavioral property, but Diamond and Stiglitz [14, p. 343] have shown that monotonicity of $-\phi_{11} / \phi_{1}$ in $\alpha$ is equivalent to a uniformly signed response of $\alpha(F)$ to compensated increases in risk. Thus, while more risk averse underlying preferences will not necessarily generate more risk averse behavior in situations of temporal risk, we at least have easily interpretable conditions under which this will happen, and in Section 4 shall examine whether these conditions are likely to be satisfied by underlying preferences in several specific cases of temporal risk.

\section{(f) Absolute and Relative Risk Aversion}

Since another important source of comparative statics results are the assumptions of decreasing absolute and increasing relative risk aversion, it is similarly important to determine whether (or when) these properties carry over from underlying preferences to preferences over temporal prospects. ${ }^{s}$ Since these properties have typically only been considered in an expected utility framework, we begin by offering the following generalizations of these behavioral characteristics. ${ }^{6}$

Definitions. The preference functional $V(\cdot)$ is said to exhibit nondecreasing (nonincreasing) absolute risk aversion if and only if the preference functional $V_{+c}(\cdot)$ defined by $V_{+c}(F) \equiv V\left(F_{+c}\right)$ is everywhere at least as risk averse as $V(\cdot)$ for all positive (negative) $c . V(\cdot)$ is said to exhibit nondecreasing (nonincreasing) relative risk aversion if and only if the preference functional $V_{\times c}(\cdot)$ defined by $V_{\times c}(F) \equiv V\left(F_{\times c}\right)$ is everywhere at least as risk averse as $V(\cdot)$ for all positive (negative) $c$.

Since comparing $Y(\cdot)$ with the preference functionals $Y_{+c}(\cdot)$ and $Y_{\times c}(\cdot)$ is equivalent to comparing the underlying utility function $\phi(\cdot, \cdot)$ with the

\footnotetext{
"For studies of when these and related properties carry over to the "timeless" preference functional $W(\cdot)$ of Section 1, see Fama $[19,20]$ and Neave [49].

${ }^{6}$ In the following definitions we abstract from the purely technical aspects introduced by the boundedness of the outcome set $[0, M]$. Note also that our definition of decreasing absolute risk aversion corresponds almost exactly to Yaari's concept of "nonincreasing total risk aversion" $\left[77\right.$, Axiom $\left.V^{\prime}\right]$ (see also [39]).
} 
functions $\phi_{+c}(\cdot, \cdot)$ (defined by $\phi_{+c}(x, \alpha) \equiv \phi(x+c, \alpha)$ ) and $\phi_{x c}(\cdot, \cdot)$ (defined by $\left.\phi_{x c}(x, \alpha) \equiv \phi(c x, \alpha)\right)$, respectively, and since $\phi(\cdot, \alpha)$ exhibits nondecreasing (nonincreasing) absolute risk aversion in $x$ if and only if $\phi_{+c}(\cdot, \alpha)$ is a concave transform of $\phi(\cdot, \alpha)$ for all $c>(<) 0$, the following result, along with its relative risk aversion analogue, follows from Theorem 8.

THEOREM 9. If $A$ is an interval in $R^{1}, \phi(\cdot, \cdot)$ is thrice continuously differentiable with $\phi_{22}(\cdot, \cdot)<0$, and $\phi(\cdot, \cdot)$ and $\alpha(\cdot)$ satisfy conditions (i) or (ii) of Theorem 8, then:

(i) If $\phi(\cdot, \alpha)$ exhibits nondecreasing (nonindecreasing) absolute risk aversion for each $\alpha \in A$, then $Y(\cdot)$ exhibits nondecreasing (nonincreasing) absolute risk aversion; and

(ii) If $\phi(\cdot, \alpha)$ exhibits nondecreasing (nonincreasing) relative risk aversion for each $\alpha \in A$, then $Y(\cdot)$ exhibits nondecreasing (nonincreasing) relative risk aversion.

(Proof in Appendix.)

In Section 4 we shall apply this result to several specific cases to get an idea of when it would and would not be realistic to assume that preferences over temporal prospects exhibit these two important aspects of behavior.

\section{(g) Comparison with Preferences over Timeless Prospects}

In this section we compare the functional $Y(\cdot)$ with the same individual's preference functional over timeless prospects, i.e., with $W(F) \equiv \int_{\alpha \in A}^{\max } \phi(x, \alpha)$ $d F(x) \equiv \int \phi(x, \alpha(x)) d F(x)$.

It is important to be precise about what this comparison involves. Referring to Fig. 4, note that the situation of timeless risk considered here differs from the situation of temporal risk we have been considering only in the timing of the choice of $\alpha$, which now may be postponed until after $\tilde{x}$ is realized. In particular, we do not consider the whole host of effects which would arise from a change in the timing of the resolution of, and hence possibly the consumption of, $\tilde{x}$ : while the effects of such a change on $\phi(\cdot, \cdot)$ are certainly worth study, they will typically depend upon the exact
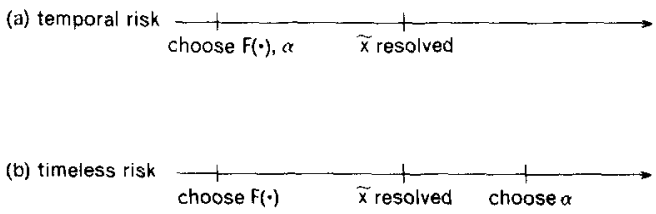

FIG. 4. Timeless/temporal risk comparison. 
circumstances of the problem and hence are beyond the scope of this paper. Note, finally, that we also assume an unchanged auxiliary choice set, postponing the effects of a change in $A$ to Section 3(h).

The temporal/timeless comparison of Fig. 4 has been examined in several previous studies, e.g., Caperaa and Eeckhoudt [10], Dreze and Modigliani [15], Eden [16], Epstein [18], Kreps and Porteus [31], Mossin [47], and Spence and Zeckhauser [68]. One of the main results in this literature is that the individual will always weakly prefer a timeless prospect to an identically distributed temporal prospects, i.e., $W(F) \geqslant Y(F)$ for all $F(\cdot)$. This may be seen to follow immediately from the definitions of $W(\cdot)$ and $Y(\cdot)$, and is sometimes described as "the expected value of perfect information can never be negative."

Another of the main results in this literature is that an individual would never assign a higher certainty equivalent (i.e., a lower risk premium) to a temporal prospect than to an identically distributed timeless prospect, or in other words, if $Y(F)=Y\left(G_{c}\right)$ and $W(F)=W\left(G_{c^{*}}\right)$, then $c \leqslant c^{*}$. Not surprisingly, this result has often been taken to imply that preferences over temporal prospects are "more risk averse" than preferences over timeless prospects. Spence and Zeckhauser [68, p. 402], for example, claim that to an external observer unaware of the distinction, an individual "will appear to be substantially more risk averse" when choosing over a set of temporal prospects than when choosing over a set of identically distributed timeless prospects. In the special case of a two-period consumption-savings model, Dreze and Modigliani [15] have similarly used risk premia for infinitesimal risks to derive "local" measures of the individual's aversion to temporal and timeless risk and have shown that the former will always be at least as great as the latter, and this result has been extended to the case of large risks by Caperaa and Eeckhoudt [10, Theorem 3].

However, since $Y(\cdot)$ is in general nonlinear, it follows that the fact that it assigns lower certainty equivalents/higher risk premia than $W(\cdot)$ is not sufficient to imply that it is everywhere more risk averse than $W(\cdot)$, and indeed this will not in general be true (an instance where $Y(\cdot)$ would demand strictly more of a risky asset than $W(\cdot)$, for example, is given in [40]). Recalling that the local utility functions of $Y(\cdot)$ and $W(\cdot)$ are $\phi(\cdot, \alpha(F))$ and $\xi(\cdot) \equiv \phi(\cdot, \alpha(\cdot))$, respectively, it follows from the Theorem of Section 2 that necessary and sufficient conditions for preferences over temporal prospects to be everywhere more risk averse than preferences over timeless prospects is given by:

THEOREM 10. The functional $Y(F) \equiv \max _{\alpha \in A} \int \phi(x, \alpha) d F(x) \equiv \int \phi(x$, $\alpha(F)) d F(x)$ will be everywhere at least as risk averse as $W(F) \equiv$ $\int \max _{\alpha \in A} \phi(x, \alpha) d F(x) \equiv \int \phi(x, \alpha(x)) d F(x)$ if and only if $\phi(x, \alpha(F))$ is at 
least as concave a function of $x$ as $\xi(x) \equiv \phi(x, \alpha(x))$ for all $F(\cdot)$ in $D[0, M]$, or equivalently, if and only if

$-\frac{\xi_{11}(x)}{\xi_{1}(x)} \equiv-\frac{\phi_{11}(x, \alpha(x))}{\phi_{1}(x, \alpha(x))}+\frac{\left(\phi_{12}(x, \alpha(x))\right)^{2}}{\phi_{1}(x, \alpha(x)) \cdot \phi_{22}(x, \alpha(x))} \leqslant-\frac{\phi_{11}(x, \alpha(F))}{\phi_{1}(x, \alpha(F))}$

for all $x \in[0, M]$ and $F(\cdot) \in D[0, M]$.

Unfortunately, while mathematically straightforward, this necessary and sufficient condition does not seem to possess an intuitive behavioral characterization.

\section{(h) Changes in the Auxiliary Choice Set}

As our final comparative statics situation we consider the effect of a change in the size of the auxiliary choice set $A$. Such a change might, for example, come from a change in the price of any good whose quantity demanded was an auxiliary decision, from a change in the individual's initial wealth level, or from a change in the timing of the auxiliary choice (e.g., where postponing an auxiliary choice led to an increase or decrease in the number of available alternatives).

One immediate result is that an expansion of the set $A$ will never make the individual worse off. In other words, defining $Y(\cdot)$ and $Y^{*}(\cdot)$ by $Y(F) \equiv$ $\max _{\alpha \in A} \int \phi(x, \alpha) d F(x)$ and $Y^{*}(F) \equiv \max _{\alpha \in A^{*}} \int \phi(x, \alpha) d F(x)$, then $Y^{*}(F) \geqslant$ $Y(F)$ for all $F(\cdot)$ whenever $A \subseteq A^{*}$.

A less transparent, though possibly more important, question is that of the effect of the size of $A$ on the individual's ordinal ranking over temporal prospects, and hence on his or her behavior in situations of temporal risk. In the most general case, it turns out that nothing at all may be said about the effect of an expansion or contraction of $A$ upon this ranking-it may become more or less risk averse, more or less quasiconcave, change from increasing to decreasing absolute risk aversion or vice versa, etc. Formally, we have:

THEOREM 11. For any continuous convex functionals $V(\cdot)$ and $V^{*}(\cdot)$ over $D[0, M]$, there exist sets $A$ and $A^{*}$, and set of functions $\{U(\cdot, \alpha) \mid$ $\left.\alpha \in A^{*}\right\}$ over $[0, M]$ such that $A \subseteq A^{*}, V(F) \equiv \max _{\alpha \in A} \int U(x, \alpha) d F(x)$, and $V^{*}(F) \equiv \max _{\alpha \in A^{*}} \int U(x, \alpha) d F(x)$.

\section{(Proof in Appendix.)}

While we can obtain no general results, we may still obtain useful results in special cases. For example, if underlying preferences are such that each function in the set $\left\{\phi(\cdot, \alpha) \mid \alpha \in A^{*}-A\right\}$ is at least as concave as each function in $\{\phi(\cdot, \alpha) \mid \alpha \in A\}$, then expanding the auxiliary choice set from $A$ to $A^{*}$ would mean that at each $F(\cdot)$ the local utility function of $Y^{*}(\cdot)$ would either be the same as that of $Y(\cdot)$ (i.e., $\phi(\cdot, \alpha(F))$ ) or else be replaced by 
some more concave function $\phi\left(\cdot, \alpha^{*}(F)\right)$ for some $\alpha^{*}(F) \in A^{*}-A$, leading to an increase in risk aversion. In the case of a univariate auxiliary choice set, therefore, we have

THeOREM 12. Let $A$ be an interval in $R^{1}$ and assume $d\left[-\phi_{11}(x, \alpha) /\right.$ $\left.\phi_{1}(x, \alpha)\right] / d \alpha \geqslant 0$ for all $(x, \alpha)$. Then, if $Y(F) \equiv \max _{\alpha \in[c, d]} \int \phi(x, \alpha) d F(x)$ and $Y^{*}(F) \equiv \max _{\alpha \in\left[c^{*}, d^{*}\right\}} \int \phi(x, \alpha) d F(x)$, then $Y^{*}(\cdot)$ will be everywhere at least as risk averse as $Y(\cdot)$ whenever $c \leqslant c^{*}$ and $d \leqslant d^{*}$.

\section{(i) Nonexpected Utility Underlying Preferences}

In light of the growing empirical evidence that individuals' underlying preferences do not satisfy the independence axiom of expected utility theory (see Section 5 for references), it is useful to consider how the above analysis extends to the case of nonexpected utility underlying preferences. While a full formal treatment is beyond the scope of this paper, we offer the following informal remarks on this topic.

Dropping the assumption that underlying preferences satisfy the independence axion implies that we replace the linear maximand $\int \phi(x, \alpha) d F(x)$ over $D[0, M] \times A$ with the nonlinear maximand $V(F, \alpha)$, which we assume to be Fréchet differentiable with local utility function $U(x ; F, \alpha)$ and with $d V(F, \alpha) / d \alpha$ denoted by $V_{2}(F, \alpha){ }^{7}$ Thus, for example, risk aversion of underlying preferences is equivalent to concavity of $U(x ; F, \alpha)$ in $x$ for all $F(\cdot)$ and $\alpha$. In such a case, it is clear that the individual's induced preference functional over temporal prospects is given by $Y^{\dagger}(F) \equiv \max _{\alpha \in A} V(F, \alpha) \equiv$ $V\left(F, \alpha^{\dagger}(F)\right)$, where $\alpha^{\dagger}(F) \equiv \operatorname{argmax}_{\alpha \in A} V(F, \alpha)$.

It is clear that the differentiability and hence continuity of $V(F, \alpha)$ ensures that $Y^{\dagger}(\cdot)$ will remain continuous, however, since $Y^{\dagger}(\cdot)$ is no longer the pointwise maximum of a set of linear functionals, it will not necessarily be quasiconvex. Graphically, this says that while the $Y^{\dagger}(\cdot)=k$ indifference curve will still be the lower envelope of the $V(\cdot, \alpha)=k$ indifference curves as $\alpha$ ranges over $A$, the fact that these latter curves will not be linear implies that the former curve need not be convex. Similarly, the loss of the linear underlying structure also means that our earlier recoverability results (Theorems 2 and 3 ) do not carry over.

However, just as the Wong-Viner-Samuelson envelope theorem may be applied to the lower envelope of a set of nonlinear short run cost curves, it is clear that, provided $A$ is a continuum, the nonlinearity of the underlying indifference curves will not affect our earlier "envelope result" (Theorem 5;

\footnotetext{
"An important example of such a nonlinear functional is the "temporal von NeumannMorgenstern" or "ordinal certainty equivalent" functional $V(F, \alpha) \equiv C\left(\alpha, \int u(x, \alpha) d F(x)\right)$ of Kreps and Porteus [31] (see also [29] and [30]) and Selden [66]. For an analysis of induced preferences when underlying preferences take this form, see [31, Section 5$]$.
} 
see also $\left[55\right.$, p. 243]). In other words, $Y^{\dagger}(\cdot)$ will be Fréchet differentiable with local utility function $U\left(\cdot ; F, \alpha^{\dagger}(F)\right)$, i.e., with the local utility function of the "conditional preference functional" $V\left(\cdot, \alpha^{\dagger}(F)\right)$ at $F(\cdot)$. Because of this identification of the local utility functions of $Y^{\dagger}(\cdot)$ with the local utility functions of the underlying preference functionals, we also see that the properties of monotonicity, risk aversion, and third-order stochastic dominance preference will continue to carry over from underlying to induced preferences.

On the other hand, our results on comparative risk aversion, absolute and relative risk aversion, and the comparison with timeless preferences do not possess immediate generalizations. The basic reason is that while in the case of the linear underlying maximand $\int \phi(x, \alpha) d F(x)$ knowledge of the local utility functions $\{\phi(\cdot, \alpha \mid \alpha \in A\}$ implied complete knowledge of how changes in $\alpha$ affected the maximand, this is no longer true in the case of the nonlinear underlying maximand $V(F, \alpha)$, where the effect of $\alpha$ (given by $V_{2}(F, \alpha)$ ) can vary independently of the set of local utility functions $\{U(\cdot ; F, \alpha)\}$. Thus, the conditions on the local utility functions in Theorems 8 through 10 are no longer sufficient to characterize these respective aspects of behavior (this is not to say that generalizations of these theorems do not exist, only that they will have to be "deeper" than simply replacing $\phi(x, \alpha)$ by $U(x ; F, \alpha)$ in the statements of the theorems). Finally, we note that an increase in the size of $A$ will of course still make the individual better off, and the immediate generalization of Theorem 12 obtained by replacing $\phi(x, \alpha)$ by $U(x ; F, \alpha)$ in fact does hold.

\section{Applications}

In this section we apply the above results to three specific cases of temporal risk.

\section{(a) Income Risk}

The most common example of the type of temporal risk known as "income risk" is that of an individual facing a set of delayed income prospects and who in the meantime must make a current consumptionsavings decision. Letting $\zeta\left(c_{1}, c_{2}\right)$ be the von Neumann-Morgenstern utility function of the consumption stream $\left(c_{1}, c_{2}\right), \alpha$ the level of savings, $F(\cdot)$ the distribution of the delayed income prospect, $I$ the initial endowment, and $R$ the (nonstochastic) gross interest rate, such an individual will rank $(F(\cdot), \alpha)$ pairs on the basis of the maximand $\int \zeta(I-\alpha, R \alpha+x) d F(x)$. In terms of our earlier notation, therefore, we have that $\phi(x, \alpha) \equiv \zeta(I-\alpha, R \alpha+x)$ and $A=[0, I]$ if no borrowing is allowed, or else $A=[-l, I]$ if the individual may borrow up to amount $l$. By appropriate assignment of variables and 
interpretation of $\zeta(\cdot, \cdot)$, this algebraic framework may also be used to represent choice over delayed income prospects with endogenous labor supply, with advance purchase of one or more commodities, and even a $T$ period consumption-savings problem with the uncertainty of the income prospects resolved after $k<T$ periods.

Since the assumptions of Sections 3(a) thrugh 3(d) are quite plausable in such situations, it follows that induced preferences in such cases will be continuous, convex, recoverable, and differentiable, and will preserve the underlying properties of monotonicity and risk aversion. If the standard assumption of declining absolute risk aversion with respect to $c_{2}$ is made (i.e., $d\left[\zeta_{22} / \zeta_{2}\right] / d c_{2} \leqslant 0$ ), it follows that $\zeta_{222}>0$, so that underlying preferences and hence induced preferences will exhibit third-order stochastic dominance preference.

Our results on comparative, absolute, and relative risk aversion were seen to depend upon the conditions that $-\phi_{11}(x, \alpha) / \phi_{1}(x, \alpha) \equiv-\zeta_{22}(I-\alpha$, $R \alpha+x) / \zeta_{2}(I-u, R \alpha+x)$ be monotonic in $\alpha$ and $\alpha\left(G_{c}\right)$ be monotonic in $c$ in the opposite direction. Since this first condition is equivalent to a uniform sign of the expression

$$
-d\left[-\xi_{22}\left(c_{1}, c_{2}\right) / \zeta_{2}\left(c_{1}, c_{2}\right)\right] / d c_{1}+R \cdot d\left[-\zeta_{22}\left(c_{1}, c_{2}\right) / \zeta_{2}\left(c_{1}, c_{2}\right)\right] / d c_{2}
$$

for all nonnegative $c_{1}, c_{2}$, and $R$, it is clear that we require increases in $c_{1}$ and $c_{2}$ to have uniform and opposite effects on the term $-\zeta_{22} / \zeta_{2}$. In fact, precisely this condition, namely, that $-\zeta_{22} / \zeta_{2}$ is nonincreasing in $c_{2}$ and nondecreasing in $c_{1}$, has been proposed by Drezc and Modigliani [15, Section 4] and Sandmo [64, p. 592; 65, p. 354] in the two-period consumption-savings case (where it has been termed "endogenously diminishing absolute risk aversion" or "decreasing temporal risk aversion"), and by Block and Heineke [8, p. 524; 9, p. 387] for the labor supply case, and is of course automatically satisfied in the widely assumed case of an additively separable function $\zeta\left(c_{1}, c_{2}\right) \equiv f\left(c_{1}\right)+g\left(c_{2}\right)$ with $g(\cdot)$ exhibiting decreasing absolute risk aversion (see, for example, Hakansson [25], Levhari and Srinivasan [32], Phelps [51], Rothschild and Stiglitz [59], and Sandmo [63]). That $-\phi_{11} / \phi_{1}$ is nonincreasing in $\alpha$ therefore seems a plausible assumption for many situations of income risk.

Since $\alpha\left(G_{c}\right)$ maximizes $\phi(c, \alpha)$, the effect of $c$ on $\alpha\left(G_{c}\right)$ will be the same as the sign of $-\phi_{12}(c, \alpha) / \phi_{22}(c, \alpha)$, which by the concavity of $\zeta(\cdot, \cdot)$ will be the same as the sign of $\phi_{12}(c, \alpha) \equiv-\zeta_{12}(I-\alpha, R \alpha+c)+R \cdot \zeta_{22}(I-\alpha, R \alpha+c)$. However, since the standard assumption that $c_{1}$ is not inferior implies that this expression is nonpositive, we have that $\alpha\left(G_{c}\right)$ is monotonic in $c$ in the same direction as $-\phi_{11} / \phi_{1}$ is in $\alpha$, which implies that properties of comparative, absolute, and relative risk aversion on underlying preferences will in general not carry over to preferences over temporal prospects in 
typical situations of income risk. Though unfortunate, this finding is still useful, for it warns us not to place too much confidence on results which depend upon these types of assumptions (e.g., declining absolute risk aversion) in situations of choice over delayed income prospects (see also Sandmo [64, p. 595] on this point).

As a final, positive result, we note that the fact that $-\phi_{11} / \phi_{1}$ is likely to be nonincreasing in $\alpha$ coupled with Theorem 12 implies that increases in the borrowing limit $l$ will make the individual everywhere at least as risk averse over temporal prospects as before the increase. ${ }^{8}$

\section{(b) Capital Risk}

The type of temporal risk known as "capital risk" concerns the case where the auxiliary variable $\alpha$ represents the "amount invested" in the chosen prospect $F(\cdot)$. In other words, using the notation of the previous section, the individual will now rank $(F(\cdot), \alpha)$ pairs on the basis of $\int \zeta(I-\alpha, \alpha x) d F(x)$, so that in this case $\phi(x, \alpha) \equiv \zeta(I-\alpha, \alpha x)$. Besides the obvious application to induced preferences over "rate of return" distributions, this algebraic formulation may also be applied to the case of labor supply with an uncertain wage, advance ordering of a commodity with uncertain price, and as in the previous section, the multi-period extension of the original twoperiod case.

As with income risk, it is likely that induced preferences here will be continuous, convex, recoverable, and differentiable, and preserve underlying properties of monotonicity and risk aversion, and, if applicable, third order stochastic dominance preference. Assuming that $\zeta(\cdot, \cdot)$ is concave, the effect of $c$ upon $\alpha\left(G_{c}\right)$ is given by the sign of $\phi_{12}(c, \alpha) \equiv \zeta_{2}(I-\alpha, \alpha x)-$ $\alpha \zeta_{12}(I-\alpha, \alpha x)+\alpha x \zeta_{22}(I-\alpha, \alpha x)$. It is straightforward to show (e.g., Sandmo $[64$, Eq. (12)]) that positivity of this term is equivalent to savings being an increasing function of the interest rate, or in our other cases, that labor supply be increasing in the wage or that the amount of the commodity ordered be decreasing in its price. That $\alpha\left(G_{c}\right)$ is increasing in $c$ therefore seems highly plausible.

The effect of $\alpha$ on $-\phi_{11}(x, \alpha) / \phi_{1}(x, \alpha)\left(\equiv-\alpha \xi_{22}(I-\alpha, \alpha x) / \zeta_{2}(I-\alpha, \alpha x)\right)$ is given by the sign of

$$
-d\left[-c_{2} \zeta_{22}\left(c_{1}, c_{2}\right) / \zeta_{2}\left(c_{1}, c_{2}\right)\right] / d c_{1}+x \cdot d\left[-c_{2} \zeta_{22}\left(c_{1}, c_{2}\right) / \zeta_{2}\left(c_{1}, c_{2}\right)\right] / d c_{2} .
$$

In this case where $\zeta\left(c_{1}, c_{2}\right)$ is separable, the first term in (4) will be zero and the standard assumption of increasing relative risk aversion in $c_{2}$ implies that the second term will be positive. In the nonseparable case, we recall from the

\footnotetext{
${ }^{8}$ The reason why Theorem 12 does not predict the same for a change in $I$ is that changes in $I$ change the definition of $\phi(x, \alpha)$ for a given $\xi(\cdot, \cdot)$.
} 
previous section that the first term will presumably be negative (i.e., the derivative will be positive), which of course would offset the positivity of the second term. However, in situations where we might expect the "own effect" of $c_{2}$ on $-c_{2} \zeta_{22} / \zeta_{2}$ to be stronger than the "cross effect" of $c_{1}$ on this term, and where the gross rate of return $x$ was always at least unity, we might retain the conclusion of the separable case, namely, that (4) is positive and hence that $-\phi_{11} / \phi_{1}$ is nondecreasing in $\alpha$. Thus, while we should probably not be as confident about our assumptions here as in the case of income risk, we nevertheless must conclude that at least the most plausible sets of assumptions on underlying preferences once again imply that the properties of comparative, absolute, and relative risk aversion on underlying preferences will in general not carry over.

\section{(c) Multiple Risky Decisions}

A third type of temporal risk is where the auxiliary decision itself consists of a risky choice, e.g., where besides choosing over delayed income prospects the individual has to make some other risky consumption-savings, real investment, or insurance decision in the meantime. In the (presumably typical) case where the two types of risks are stochastically independent and additive, we may represent the induced preference functional (over $\tilde{x}$ distributions) as $\quad Y\left(F_{\tilde{x}}\right) \equiv \max _{\alpha \in A} \iint v(x+y) d F_{\tilde{y}}(y ; \alpha) d F_{\tilde{x}}(x)$, where $\left\{F_{\tilde{y}}(\cdot ; \alpha) \mid \alpha \in A\right\}$ is the set of alternative distributions of the auxiliary random variable $\tilde{y}$ and $v(\cdot)$ is the von Neumann-Morgenstern utility of total wealth function. Since this implies that $\phi(x, \alpha) \equiv \int v(x+y) d F_{\tilde{y}}(y ; \alpha)$, it follows immediately that $\phi_{1}, \phi_{11}$, and $\phi_{111}$ will take on the respective signs of $v^{\prime}, v^{\prime \prime}, v^{\prime \prime \prime}$, so that monotonicity, risk aversion, and, if applicable, third-order stochastic dominance preference will carry over from $v(\cdot)$ to induced preferences here (see Hadar and Russell [23], Tesfatsion [70], and Levy and Kroll [33] on this point). Similarly, Pratt [52, Theorem 5] has shown that the property of decreasing absolute risk aversion (though not increasing relative risk aversion) will carry over from $v(\cdot)$ to $\phi(x, \alpha)$ for each $\alpha$, and Kihlstrom et al. [28, p. 916] have shown that the property of comparative risk aversion will carry over from a pair of $v(\cdot)$ functions to the corresponding $\phi(\cdot, \alpha)$ functions provided at least one of the $v(\cdot)$ 's exhibits decreasing absolute risk aversion.

However, it is impossible to determine whether the properties of comparative and declining absolute risk aversion will carry over from $\phi(\cdot, \cdot)$ to induced preferences without more fully specifying the nature of the function $\phi(\cdot, \cdot)$, and in particular the nature of the auxiliary set $\left\{F_{\tilde{y}}(\cdot ; \alpha) \mid \alpha \in A\right\}$ which generates it. One presumably common case would be where the set $\left\{F_{\tilde{y}}(\cdot ; \alpha) \mid \alpha \in A\right\}$ represents the "efficient set" of some set of distributions, so that changes in $\alpha$ moved the distribution $F_{\tilde{y}}(\cdot ; \alpha)$ up along some "risk-return frontier." While there exist many possible specifications of 
such a risk-return tradeoff (e.g., Diamond and Stiglitz [14], Ross [56]), we consider the simple case where $F_{\tilde{y}}(\cdot ; \alpha)$ is the distribution of the random variable $r+\alpha \tilde{z}$ with $r>0$ and $\tilde{z}$ a random variable with positive mean but with possible negative values. In this case we have $\phi(x, \alpha) \equiv \int v(x+r+\alpha z)$ $d F_{\tilde{z}}(z)$, where $F_{\tilde{z}}(\cdot)$ is the distribution of $\tilde{z}$, and Arrow [5] has shown that $d a\left(G_{c}\right) / d c$ will be positive provided $v(\cdot)$ exhibits decreasing absolute risk aversion in the Arrow-Pratt sense. Similarly, the sign of $d\left[-\phi_{11} / \phi_{1}\right] / d \alpha$ is given by the sign of

$$
\begin{aligned}
-\iint & \left\{\hat { z } \left[v^{\prime}(x+r+\alpha z) v^{\prime \prime \prime}(x+r+\alpha \hat{z})\right.\right. \\
& \left.\left.-v^{\prime \prime}(x+r+\alpha z) v^{\prime \prime}(x+r+\alpha \hat{z})\right]\right\} d F_{\tilde{z}}(z) d F_{\tilde{z}}(\hat{z}) .
\end{aligned}
$$

If $v(\cdot)$ is concave, then nonnegativity of the term in square brackets in (4) is equivalent to the Ross [56] characterization of decreasing absolute risk aversion, which, while stronger than the Arrow-Pratt characterization, is nevertheless still a plausible assumption on preferences. However, since $\tilde{z}$ takes on both positive and negative values, the sign of (4), and hence of $d\left[-\phi_{11} / \phi_{1}\right] / d \alpha$, will be indeterminate. Thus, as in the cases of income and capital risk, while results depending on monotonicity, risk aversion, and third order stochastic dominance preference will be robust to the existence of other risky decisions, results depending on comparative, absolute, and relative risk aversion will probably not be.

\section{CONCLUSION: INDUCED VS OBSERVED VIOLATIONS OF THE INDEPENDENCE AXIOM}

In Section 3(i) we referred to experimental findings that individual preferences over risky prospects pervasively and systematically violate the independence axiom of expected utility theory, or in other words, are systematically nonlinear in the probabilities. These studies, which have been conducted over several years, by researchers in various fields, and using several different approaches, have uncovered four "effects":

The "common consequence effect,"which includes the Allais Paradox [2, p. 89] as a special case, and which has been observed by Allais [3], Hagen [24], Kahneman and Tversky [26], MacCrimmon [36], MacCrimmon and Larsson [37], Morrison [45], Moskowitz [46], Raiffa [54], and Slovic and Tversky [67].

The "common ratio effect," which includes the "certainty effect" [26] and "Bergen Paradox" [24] as special cases, and which has been observed by Hagen [24], Kahneman and Tversky [26], MacCrimmon and Larsson [37], and Tversky [73]. 


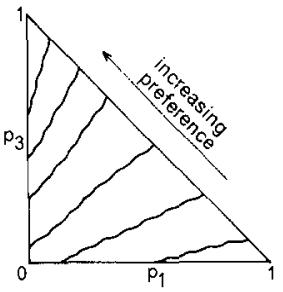

FIG. 5. The "fanning out" of indifference curves implied by Hypothesis II.

"Oversensitivity to changes in the probabilities of small probabilityoutlying events" [38], which may be uncovered from empirical fittings of nonlinear preference functionals by Ali [1], Edwards [17], Griffith [22], Nogee and Leibermann [50], Preston and Baratta [53], Sprowls [69], and Tversky $[71,72]$.

The "utility evaluation effect" [41], observed by Karmarkar [27] and McCord and de Neuville [43], and which can also be recovered from the evidence in Allais [3].

(For surveys of this literature, see MacCrimmon and Larsson [37] and Machina [38, 41].)

In [38] and [41] it was shown that each of these types of behavior will follow from a single assumption on the shape of the individual preference functional $V(\cdot)$, so that individuals observed to be violating the independence axiom are evidently acting much more consistently and systematically than had been supposed. ${ }^{9}$ This assumption, expressed in terms of the local utility functions $\{U(\cdot ; F)\}$ of $V(\cdot)$, is given by:

HYPOTHESIS II [38]. $-U_{11}\left(x ; F^{*}\right) / U_{1}\left(x ; F^{*}\right) \geqslant-U_{11}(x ; F) / U_{1}(x: F)$ for all $x \in[0, M]\left(\right.$ i.e., $U\left(\cdot ; F^{*}\right)$ is more concave than $U(\cdot ; F)$ ) whenever $F^{*}(\cdot)$ first-order stochastically dominates $F(\cdot)$.

In terms of the triangle diagram of Sections 2 and 3, Hypothesis II implies that indifference curves "fan out" as illustrated in Fig. 5 (see [38] and [41] for more detail).

Because this specific form of nonlinearity has been so widely observed, and is not without policy implications [41, Section IV.F], it is natural to ask in which circumstances induced preferences will satisfy Hypothesis II. The answer is given by:

${ }^{9}$ See Weber [75], however, for a discussion of some observed auction behavior which is evidently not compatible with Hypothesis II below. 
THEOREM 13. If $\phi(\cdot, \cdot)$ is thrice continuously differentiable with $\phi_{22}(\cdot, \cdot)<0$ and $\phi(\cdot, \cdot)$ and $\alpha(\cdot)$ satisfy either of the (equivalent) conditions

(i) $\phi_{12}(x, \alpha)$ and $d\left[-\phi_{11}(x, \alpha) / \phi_{1}(x, \alpha)\right] / d \alpha$ are either both everywhere nonnegative or else everywhere nonpositive for all $x \in[0, M]$, and $\alpha \in\{\alpha(F) \mid F(\cdot) \in D[0, M]\}$, or

(ii) either $\alpha\left(F^{*}\right) \geqslant \alpha(F)$ and $\alpha\left(F^{* * *}\right) \leqslant \alpha\left(F^{* *}\right)$ whenever $F^{*}(\cdot)$ first order stochastically dominates $F(\cdot)$ and $F^{* * *}(\cdot)$ differs from $F^{* *}(\cdot)$ by a compensated increase in risk, or else $\alpha\left(F^{*}\right) \leqslant \alpha(F)$ and $\alpha\left(F^{* * *}\right) \geqslant \alpha\left(F^{* *}\right)$ for all such $F(\cdot), F^{*}(\cdot), F^{* *}(\cdot)$, and $F^{* * *}(\cdot)$, then $Y(F) \equiv$ $\max _{\alpha \in A} \int \phi(x, \alpha) d F(x) \equiv \int \phi(x, \alpha(F)) d F(x)$ satisfies Hypothesis II.

\section{(Proof in Appendix.)}

In Section 4 we saw that standard assumptions on underlying preferences in situations of income risk implied that both $\phi_{12}$ and $d\left[-\phi_{11} / \phi_{1}\right] / d \alpha$ were nonpositive, which implies condition (i) of this theorem. Similarly, although there was more ambiguity in the case of capital risk, the "most plausible" case was that these two terms were nonnegative, which also implies condition (i). Accordingly, we should expect induced preferences in almost all situations of income risk and most cases of capital risk to exhibit precisely the type of nonlinearity revealed in the above four observed "effects."

Should we regard this as an explanation of these empirical findings? Probably not: while it is possible that subjects might have viewed the risky prospects of the experiments as delayed, the implicit (and often explicit) assumption was undoubtedly that they were to be immediately resolved. Our conclusion therefore would seem to be that the existence of temporal risk and the nature of underlying preferences are two separate sources of the types of behavior captured by Hypothesis II, which suggests the importance of further research into the economic implications of this property of preferences.

Related work. Besides the important early work of Markowitz [42], Mossin [47], Spence and Zeckhauser [68], and Dreze and Modigliani [15], the reader is referred to the more recent and complementary analyses of Epstein [18], Kreps and Porteus [31], and Rossman and Selden [57].

\section{APPENDIX}

Proof of Theorem 2. If $V\left(F^{*}\right)<\sup _{U \in Z^{*}} \int U(x) d F^{*}(x)$ for some $F^{*}(\cdot)$, then there would exist some $U^{*}(\cdot) \in Z^{*}$ for which $V\left(F^{*}\right)<\int U^{*}(x) d F^{*}(x)$, contradicting the definition of $Z^{*}$. 
Alternatively, assume that there exists $F^{*}(\cdot)$ such that $V\left(F^{*}\right)>\int U(x)$ $d F^{*}(x)$ for all $U(\cdot) \in Z^{*}$. Since $V(\cdot)$ is continuous and convex, the set $\Phi=$ $\{(F, \alpha) \mid V(F)<\alpha\}$ is open and convex. Since $\left(F^{*}, V\left(F^{*}\right)\right) \notin \Phi$, we have from the separating hyperplane theorem (Rudin 60, p. 58]) that there will exist a continuous linear $\Lambda(\cdot, \cdot)$ such that $0=\Lambda\left(F^{*}, V\left(F^{*}\right)\right)>\Lambda(F, \alpha)$ for all $(F, \alpha) \in \Phi$. Without loss of generality, we can represent $\Lambda(F, \alpha)$ by $\int U^{*}(x) d F(x)-\alpha$ for some continuous $U^{*}(\cdot)$, so that we have $0=\int U^{*}(x) d F^{*}(x)-V\left(F^{*}\right)>\int U^{*}(x) d F(x)-\alpha$ for all $F(\cdot)$ and $\alpha>V(F)$. This implies that $\int U^{*}(x) d F(x) \leqslant V(F)$ for all $F(\cdot)$, so that $U^{*}(\cdot) \in \Phi$. The fact that $V\left(F^{*}\right)=\int U^{*}(x) d F^{*}(x)$ therefore contradicts the initial assumption of this paragraph. Note, finally, that the above arguments also establish that $\int U(x) d F(x)$ will always attain its maximum over $U(\cdot) \in Z^{*}$.

Proof of Theorem 3. Since $Z^{*}$ is clearly closed, convex, comprehensive, and contains $Z$, it follows that $Z^{*}$ contains the convex comprehensive closure of $Z$. Assume now that $\hat{U}(\cdot)$ is not in the convex comprehensive closure of $Z$. By Rudin [60, Theorem 3.4] and Luenberger [35, p. 113] there will exist a signed measure $\mu$ over $[0, M]$ such that $\int \hat{U}(x) d \mu(x)>\gamma_{1}>\gamma_{2}>$ $\int U(x) d \mu(x)$ for all $U(\cdot)$ in the convex comprehensive closure of $Z$. By comprehensiveness, it is clear that $\mu$ is a proper (i.e., nonnegative) measure, so we may normalize to get that there exists some $\hat{F}(\cdot) \in D[0, M]$ such that $\int \hat{U}(x) d \hat{F}(x)>\gamma_{1}^{\prime}>\gamma_{2}^{\prime}>\int U(x) d \hat{F}(x)$ for all $U(\cdot)$ in the convex comprehensive closure of $Z$. Since $Z$ is contained in its convex comprehensive closure, this implies that $\int \hat{U}(x) d \hat{F}(x)>\gamma_{1}^{\prime}>\gamma_{2}^{\prime} \geqslant V(\hat{F})$, which implies that $\hat{U}(\cdot)$ cannot be an element of $Z^{*}$.

Proof of Theorem 4. The first sentence of the statement of the theorem follows immediately from the strict monotonicity and continuity of $\phi(x, \alpha)$ in $x$. Defining $\underline{u}=\max _{\alpha \in A} \phi(0, \alpha)$ and $\bar{u}=\max _{\alpha \in A} \phi(M, \alpha)$, we have that the function $p(F)$ is defined implicitly by $Y(F) \equiv(1-p(F)) \underline{u}+p(F) \bar{u}$, so that solving gives $p(F) \equiv(Y(F)-\underline{u}) /(\bar{u}-\underline{u})$.

Proof of Theorem 5. (See [38, pp. 293-294; 60, p. 248], as well as the related result of Kreps and Porteus [31, p. 92].) Given an arbitrary $\varepsilon>0$, upper semicontinuity of $\alpha(\cdot)$ at $F_{0}(\cdot)$ and continuity of $Y(\cdot ; \alpha)$ in $\alpha$ imply that there exists a $\delta>0$ such that $\left\|F_{1}-F_{0}\right\|<\delta$ and $\alpha \in \alpha\left(F_{1}\right)$ implies $\sup _{F, F^{*} \in D[0, M]} \mid Y\left(F^{*}-F ; \alpha_{1}\right)-Y\left(F^{*}-F ; \alpha\left(F_{0}\right)\right) /\left\|F^{*}-F\right\|<\varepsilon$. Now, for any $F_{1}(\cdot)$ and $\alpha_{1} \in \alpha\left(F_{1}\right)$ we have $\left[Y\left(F_{1}\right)-Y\left(F_{0}\right)-\int \phi\left(x, \alpha\left(F_{0}\right)\right)\left[d F_{1}(x)-\right.\right.$ $\left.\left.d F_{0}(x)\right]\right] /\left\|F_{1}-F_{0}\right\|=\left[Y\left(F_{1}\right)-\int \phi\left(x, \alpha\left(F_{0}\right)\right) d F_{1}(x)\left\|F_{1}-F_{0}\right\|\right.$, which is nonnegative by definition of $Y(\cdot)$. Similariy, $\left\|F_{1}-F_{0}\right\|<\delta$ and $\alpha_{1} \in \alpha\left(F_{1}\right)$ implies 


$$
\begin{aligned}
& {\left[Y\left(F_{1}\right)-Y\left(F_{0}\right)-\int \phi\left(x, \alpha\left(F_{0}\right)\right)\left[d F_{1}(x)-d F_{0}(x)\right]\right] /\left\|F_{1}-F_{0}\right\| } \\
&= {\left[\int\left[\phi\left(x, \alpha_{1}\right)-\phi\left(x, \alpha\left(F_{0}\right)\right)\right]\left[d F_{1}(x)-d F_{0}(x)\right]\right.} \\
&\left.+\int\left[\phi\left(x, \alpha_{1}\right)-\phi\left(x, \alpha\left(F_{0}\right)\right)\right] d F_{0}(x)\right] /\left\|F_{1}-F_{0}\right\| \\
& \leqslant {\left[\int\left[\phi\left(x, \alpha_{1}\right)-\phi\left(x, \alpha\left(F_{0}\right)\right)\right]\left[d F_{1}(x)-d F_{0}(x)\right]\right] /\left\|F_{1}-F_{0}\right\| } \\
&= {\left[Y\left(F_{1}-F_{0} ; \alpha_{1}\right)-Y\left(F_{1}-F_{0} ; \alpha\left(F_{0}\right)\right)\right] /\left\|F_{1}-F_{0}\right\|<\varepsilon, }
\end{aligned}
$$

which establishes the required result.

Proof of Theorem 6. Parts (i) and (ii) of the theorem follow immediately from Theorem 5 and Theorems 1 and 2 of [38], and part (iii) parallels these proofs almost exactly.

Proof of Theorem 7. $Y^{*}\left(G_{c^{*}}\right)=Y^{*}(F)=\max _{\alpha \in A} \int \rho(\phi(x, \alpha)) d F(x) \leqslant$ $\max _{\alpha \in A} \rho\left(\int \phi(x, \alpha) d F(x)\right)=\rho\left(\max _{\alpha \in A} \int \phi(x, \alpha) d F(x)\right)=\rho(Y(F))=$ $\rho\left(Y\left(G_{c}\right)\right)=\rho\left(\max _{\alpha \in A} \phi(c, \alpha)\right)=\max _{\alpha \in A} \rho(\phi(c, \alpha))=Y^{*}\left(G_{c}\right)$, which, since $Y^{*}(\cdot)$ satisfies first order stochastic dominance preference, implies $c^{*} \geqslant c$.

Proof of Theorem 8. (i) $\rightarrow$ (ii) Assume $\alpha\left(G_{c}\right)$ is not weakly monotonic in $c$. Then there must exist open sets $\kappa_{1}$ and $\kappa_{2}$ in $[0, M]$ and points $c_{1}^{*} \in \kappa_{1}$ and $c_{2}^{*} \in \kappa_{2}$ such that $c_{1}^{*}<c_{1}^{*}, \alpha\left(G_{c_{1}^{*}}\right)=\alpha\left(G_{c_{2}^{*}}\right)$, and $d \alpha\left(G_{c}\right) / d c$ takes on uniform and opposite signs over $\kappa_{1}$ and $\kappa_{2}$. Let $\Delta$ be the intersection of the images of $\kappa_{1}$ and $\kappa_{2}$ under $\alpha(\cdot)$. Now, either (a) $d\left[-\phi_{11}(x, \alpha) / \phi_{1}(x, \alpha)\right] / d \alpha \neq 0$ for some $\hat{x} \in[0, M]$ and $\hat{\alpha} \in \Delta$ or else (b) this derivative is zero for all such $x$ and $\alpha$. In case (a) we have that there will exist $\hat{c}_{1} \in \kappa_{1}$ and $\hat{c}_{2} \in \kappa_{2}$ such that $\alpha\left(G_{\hat{c}_{1}}\right)=\alpha\left(G_{\hat{c}_{2}}\right)=\hat{\alpha}$ and $d\left[-\phi_{11}\left(\hat{x}, \alpha\left(G_{c}\right)\right) /\right.$ $\left.\phi_{1}\left(\hat{x}, \alpha\left(G_{c}\right)\right)\right] / d c$ takes on opposite signs at $\hat{c}_{1}$ and $\hat{c}_{2}$, contradicting (i). In case (b), there will exist some $c_{1}^{\prime} \in \kappa_{1}$ and $\alpha^{\prime} \in \Delta$ such that $c_{1}^{*}<c_{1}^{\prime}<c_{2}^{*}$ and $\alpha^{\prime}=\alpha\left(G_{c_{1}^{\prime}}\right) \neq \alpha\left(G_{c_{1}^{*}}\right)=\alpha\left(G_{c_{2}^{*}}\right)=\alpha^{*}$, which implies $\phi\left(c_{1}^{*}, \alpha^{*}\right)>\phi\left(c_{1}^{*}, \alpha^{\prime}\right)$ and $\phi\left(c_{1}^{\prime}, \alpha^{*}\right)<\phi\left(c_{1}^{\prime}, \alpha^{\prime}\right)$, so that the functions $\phi\left(\cdot, \alpha^{*}\right)$ and $\phi\left(\cdot, \alpha^{\prime}\right)$ cross between $c_{1}^{*}$ and $c_{1}^{\prime}$. However, since (b) implies that $\phi\left(\cdot, \alpha^{*}\right)$ and $\phi\left(\cdot, \alpha^{\prime}\right)$ must be cardinally equivalent, they can cross no more than once, so that $\phi\left(c_{2}^{*}, \alpha^{*}\right)<$ $\phi\left(c_{2}^{*}, \alpha^{\prime}\right)$, which is a contradiction. This establishes that $\alpha\left(G_{c}\right)$ is weakly monotonic in $c$.

Consider the case when $\alpha\left(G_{c}\right)$ is nondecreasing in $c$ (the opposite case follows similarly). Assume $d\left[-\phi_{11} / \phi_{1}\right] / d \alpha>0$ for some $\bar{x} \in[0, M]$ and $\bar{\alpha}=\alpha(\bar{F})$ for some $\bar{F}(\cdot)$. Since $\int \phi_{2}(x, \bar{\alpha}) d \bar{F}(x)=0$, this implies that $\phi_{2}(\bar{c}, \bar{\alpha})=0$ for some $\bar{c}$, or in other words that $\bar{\alpha}=\alpha\left(G_{\bar{c}}\right)$. To see that the range of $\alpha\left(G_{c}\right)$ as $c$ varies over $[0, M]$ cannot be just the singleton $\{\bar{\alpha}\}$, note 
that if this were the case we would have $\phi_{12}(\cdot, \bar{\alpha}) \equiv \phi_{112}(\cdot, \bar{\alpha}) \equiv 0$, contradicting the previous inequality. It therefore follows from Sard's theorem that there will exist some $\alpha^{*}$ (near $\bar{\alpha}$ ) and $c^{*}$ such that $\alpha^{*}=\alpha\left(G_{c^{*}}\right)$, $d\left[-\phi_{11} / \phi_{1}\right] / d \alpha>0$ at $\left(\bar{x}, \alpha^{*}\right)$, and $d \alpha\left(G_{c}\right) / d c>0$ at $c^{*}$. This implies $d\left[-\phi_{11}\left(\bar{x}, \alpha\left(G_{c}\right)\right) / \phi_{1}\left(\bar{x}, \alpha\left(G_{c}\right)\right)\right] / d c>0$ at $c^{*}$, contradicting (i).

(ii) $\rightarrow$ (iii) We consider the case when $\rho(\cdot)$ is concave (the opposite case follows similarly). From the Corollary to Theorem 4 of Diamond and Stiglitz [14, p. 351] it follows that $\alpha\left(G_{c}\right)$ nondecreasing (nonincreasing) in $c$ implies that $\alpha^{*}(F)=\operatorname{argmax}_{\alpha \in A} \int \rho(\phi(x, \alpha)) d F(x) \leqslant(\geqslant) \alpha(F)$. Since the monotonicity of $-\phi_{11} / \phi_{1}$ in $\alpha$ is in the opposite direction of the monotonicity of $\alpha\left(G_{c}\right)$ in $c$, it follows that, defining $\phi^{*}(x, \alpha) \equiv \rho(\phi(x, \alpha))$, we have $-\phi_{11}^{*}(x, \alpha(F)) / \phi_{1}^{*}(x, \alpha(F)) \geqslant-\phi_{11}(x, \alpha(F)) / \phi_{1}(x, \alpha(F))$ for all $x$ and $F(\cdot)$, extablishing the required result.

(iii) $\rightarrow$ (i) Assume $d\left[-\phi_{11}\left(x, \alpha\left(G_{c}\right)\right) / \phi_{1}\left(x, \alpha\left(G_{c}\right)\right)\right] / d c>0$ for some $(\bar{x}, \bar{c})$. This implies $\operatorname{sgn}\left(d\left[-\phi_{11} / \phi_{1}\right] / d \alpha\right)=\operatorname{sgn}\left(d \alpha\left(G_{c}\right) / d c\right) \neq 0$ at $\left(\bar{x}, \alpha\left(G_{\bar{c}}\right)\right)$ and $\bar{c}$, respectively. Without loss of generality, assume both derivatives are positive. $d \alpha\left(G_{c}\right) / d c$ positive at $\bar{c}$ implies $\phi_{2}(\bar{c}, \bar{\alpha})=0$ and $\phi_{12}(\bar{c}, \bar{\alpha})>0$, where $\bar{\alpha}=\alpha\left(G_{\bar{c}}\right)$, which implies there exist distinct $c_{1}$ and $c_{2}$ and uniform distribution $\bar{F}(\cdot)$ over $\left[c_{1}, c_{2}\right]$ such that $\int \phi_{2}(x, \bar{\alpha}) d \bar{F}(x)=0$ and $\phi_{12}(\cdot, \bar{\alpha})>0$ over $\left[c_{1}, c_{2}\right]$, so that $\bar{\alpha}=\alpha(\bar{F})$ as well. It therefore follows from the proof of Diamond and Stiglitz's Theorem $4[14$, p. 349] that for any arbitrarily small nonzero decrease in $\alpha$ from $\bar{\alpha}=\alpha(\bar{F})$ there will exist some $\rho(\cdot)$ with $\rho^{\prime \prime}(\cdot)<0$ over $\left[c_{1}, c_{2}\right]$ and $\rho^{\prime \prime}(\cdot)=0$ over $[0, M]-\left[c_{1}, c_{2}\right]$ such that replacing $\phi(\cdot, \cdot)$ by $\rho(\phi(\cdot, \cdot))$ induces the optimal $\alpha$ to decrease from $\alpha(\bar{F})$ by this amount. This implies that there will exist some (increasing) concave $\rho(\cdot)$ such that $-\phi_{11}^{*}\left(\bar{x}, \alpha^{*}(\bar{F})\right) / \phi_{1}^{*}\left(\bar{x}, \alpha^{*}(\bar{F})\right)<-\phi_{11}(\bar{x}, \alpha(\bar{F})) / \phi_{1}\left(\bar{x}, \alpha(\bar{F})\right.$ ) (where $\phi^{*}(\cdot, \cdot) \equiv$ $\rho(\phi(\cdot, \cdot))$ and $\alpha^{*}(\bar{F})=\operatorname{argmax}_{\alpha \in A} \int \phi^{*}(x, \alpha) d \bar{F}(x)$, which contradicts (iii).

Proof of Theorem 9. (i) Defining $\alpha_{+c}(F)=\operatorname{argmax}_{\alpha \in A} \int \phi_{+c}(x, \alpha) d F(x)$ and noting that the local utility function of $V_{+c}(\cdot)$ at $F(\cdot)$ is $\phi_{+c}\left(x, \alpha_{+c}(F)\right) \equiv \phi\left(x+c, \alpha_{+c}(F)\right)$, we see that we need to establish that $\quad-\phi_{11}\left(x+c, \alpha_{+c}(F)\right) / \phi_{1}\left(x+c, \alpha_{+c}(F)\right) \geqslant(\leqslant)-\phi_{11}(x, \alpha(F)) / \phi_{1}(x, \alpha(F))$ for each $x, F(\cdot)$, and $c>0$. Since $\phi(\cdot, \alpha)$ exhibits nondecreasing (nonincreasing) absolute risk aversion in $x$ for each $\alpha$, it suffices to establish that $-\phi_{11}\left(x, \alpha_{+c}(F)\right) / \phi_{1}\left(x, \alpha_{+c}(F)\right) \geqslant(\leqslant)-\phi_{11}(x, \alpha(F)) / \phi_{1}(x, \alpha(F))$ for each $x$ and $F(\cdot)$. The proofs of Diamond and Stiglitz's Theorem 4 [14, p. 349] and Theorem 8 above may then be adapted to obtain this result.

A similar argument applies to (ii).

Proof of Theorem 11. Let $\{\hat{U}(\cdot, \alpha) \mid \alpha \in A\}$ and $\left\{\hat{U}(\cdot, \alpha) \mid \alpha \in A^{\prime}\right\}$ be the sets from Theorem 2 which satisfy $Y(F) \equiv \max _{\alpha \in A} \int \hat{U}(x, \alpha) d F(x)$ and $Y^{*}(F) \equiv \max _{\alpha \in A^{\prime}} \int \hat{U}(x, \alpha) d F(x)$, where without loss of generality we may assume that the sets $A$ and $A^{\prime}$ are distinct and that $\hat{U}(\cdot, \cdot) \geqslant 0$. Defining $A^{*}$ 
as the union of $A$ and $A^{\prime}, T$ as a number greater than the highest value of $\hat{U}(x, \alpha)$ over $[0, M] \times A$, and $U(x, \alpha)$ to equal $\hat{U}(x, \alpha)$ over $[0, M] \times A$ and $\hat{U}(x, \alpha)+T$ over $[0, M] \times\left(A^{*}-A\right)=[0, M] \times A^{\prime}$ then yields the desired result.

Proof of Theorem 13. (i) $\leftrightarrow$ (ii) That $\phi_{12}(x, \alpha)$ will be nonnegative (nonpositive) for all such $(x, \alpha)$ if and only if first order stochastically dominating shifts in $F(\cdot)$ not lower (not raise) $\alpha(F)$ follows immediately from the first-order condition $\int \phi_{2}(x, \alpha(F)) d F(x)=0$. The relationship between $d\left[-\phi_{11} / \phi_{1}\right] / d \alpha$ and the effects of a compensated increase in risk on $\alpha(F)$ follow from Theorem 2 of Diamond and Stiglitz [14, p. 243].

(i) $\rightarrow$ (iii) If $\phi_{12}(\cdot, \cdot)$ and $d\left[-\phi_{11} / \phi_{1}\right] / d \alpha$ are both nonnegative (nonpositive) then a first order stochastically dominating shifts in $F(\cdot)$ will not lower (not raise) $\alpha(F)$, which therefore will not lower $-\phi_{11} / \phi_{1}$ for all $x$.

\section{REFERENCES}

1. M. M. All, Probability and utility estimates for racetrack bettors, J. Polit. Econ. 85 (1977), 803-815.

2. M. Allais. The foundations of a positive theory of choice involving risk and a criticism of the postulates and axioms of the American School, in [4].

3. M. Allais, The so-called Allais Paradox and rational decisions under uncertainty, in $|4|$.

4. M. Allais and O. Hagen (Eds.), "Expected Utility Hypotheses and the Allais Paradox," Reidel, Dordrecht, 1979.

5. K. J. Arrow, "Essays in the Theory of Risk-Bearing," North-Holland, Amsterdam, 1974.

6. K. J. ARrow AND F. H. HAHN, "General Competitive Analysis," Holden-Day, San Francisco, 1971.

7. P. Billingsley, "Convergence of Probability Measures," New York, 1968.

8. M. K. Block AND J. M. HeINEKe, A comment on uncertainty and household decisions, Rev. Econ. Stud. 39 (1972), 325-525.

9. M. K. Block AND J. M. Heineke, The allocation of effort under uncertainty: The case of risk averse behavior, J. Polit. Econ. 81 (1973), 376-385.

10. P. CaperaA AND L. Eeckhoudt, Delayed risks and risk premiums, J. Finan. Econ, 2 (1975), 309-320.

11. M. Chacholiades. "International Trade Theory and Policy," McGraw-Hill, New York, 1978.

12. S. H. CHEw, A generalization of the quasilinear mean with applications to the measurement of income inequality and decision theory resolving the Allais Paradox, Econometrica 51 (1983), 1065-1092.

13. J. C. Cox, Properties of functions which are solutions to maximization problems, J. Econ. Theory 6 (1973), 396-398.

14. P. A. Diamond and J. E. Stiglitz, Increases in risk and in risk aversion, J. Econ. Theory 8 (1974), 337-360.

15. J. H. Dreze and F. Modiglian, Consumption decisions under uncertainty, J. Econ. Theory 5 (1972), 308-335.

16. B. EDEN, The role of insurance and gambling in allocating risk over time, J. Econ. Theory 16 (1977), 228-246. 
17. W. Edwards, The prediction of decisions among bets, J. Exp. Psychol. 50 (1955), 201-214.

18. L. G. EPSTEIN, Decision making and the temporal resolution of uncertainty, Int. Econ. Rev. 21 (1980), 269-283.

19. E. F. Fama, Multiperiod consumption-investment decisions, Amer. Econ. Rev. 60 (1970), 163-174.

20. E. F. Fama, Multiperiod consumption-investment decisions: A correction, Amer. Econ. Rev. 66 (1976), 723-724.

21. J. M. Grandmont, Temporary general equilibrium theory, Econometrica 45 (1977), $535-572$.

22. R. M. GRIFFith, Odds adjustment by American horse-race bettors, Amer. J. Psychol. 62 (1949), 290-294.

23. J. Hadar and W. R. Russell, Stochastic dominance and diversification, J. Econ. Theory 3 (1971), 288-305.

24. O. HAGEN, Towards a positive theory of preferences under risk, in [4].

25. N. H. HaKansson, Optimal investment and consumption strategies under risk for a class of utility functions, Econometrica 38 (1970), 587-607.

26. D. Kahneman and A. TVersky, Prospect theory: An analysis of decision under risk, Econometrica 47 (1979), 263-291.

27. U. Karmarkar, "The Effect of Probabilities on the Subjective Evaluation of Lotteries," Massachusetts Institute of Technology Sloan School of Management Working Paper No. 698-74, 1974.

28. R. E. Kinlstrom, D. Romer, and S. Williams, Risk aversion with random initial wealth, Econometrica 49 (1981), 911-920.

29. D. M. Kreps and E. L. Porteus. Temporal resolution of uncertainty and dynamic choice theory, Econometric 46 (1978), 185200.

30. D. M. Kreps and E. L. Porteus, Dynamic choice theory and dynamic programming, Econometrica 47 (1979), 91-100.

31. D. M. KREPS aND E. L. Porteus, Temporal von Neumann-Morgenstern and induced preferences, J. Econ. Theory 20 (1979), 81-109.

32. D. Levhari and T. N. Srinivasan, Optimal savings under uncertainly, Rev, Econ. Stud. 36 (1969), 153-163.

33. H. LEVY AND Y. KROLL, Investment decision rules, diversification, and the investor's initial wealth, Econometrica 46 (1978), 1231-1237.

34. N. Liviatan, The principle of two stage maximization in price theory, in "Value. Capital, and Growth" (J. N. Wolfe, Ed.), Aldine, Chicago, 1968.

35. D. G. Luenderger, "Optimization by Vector Space Methods," Wiley, New York, 1969.

36. K. R. MACCRIMmon, Descriptive and normative implications of the decision-theory postulates, in "Risk and Uncertainty: Proceedings of a Conference Held by the International Economic Association" (K. Borch and J. Mossin, Eds.). Macmillan \& Co., London, 1968.

37. K. R. MacCrimmon and S. Larsson, Utility theory: Axioms versus "paradoxes," in [4].

38. M. J. MACHiNA, "Expected utility" analysis without the independence axiom, Econometrica 50 (1982), 277--323.

39. M. J. MACHINA, A stronger characterization of declining risk aversion, Econometrica $\mathbf{5 0}$ (1982), 1069-1079.

40. M. J. MaChinA, Flexibility and the demand for risky assets, Econ. Lett. 10 (1982), $71-76$.

41. M. J. Machina, Generalized expected utility analysis and the nature of observed violations of the independence axiom, in "Foundations of Utility and Risk Theory with Applications” (B. Stigum and F. Wenstøp, Eds.), Reidel, Dordrecht, 1983. 
42. H. Markowitz, "Portfolio Selection: Efficient Diversification of Investments," Yale Univ. Press, New Haven, Conn., 1959.

43. M. MCCORD AND R. DE Neufville, Fundamental deficiencies of expected utility decision analysis, unpublished manuscript, Massachusetts Institute of Technology, 1982.

44. F. MiLne, Induced preferences and the theory of the consumer, J. Econ. Theory 24 (1981), 205-217.

45. D. G. Morrison, On the consistency of preferences in Allais' Paradox, Behav. Sci. 12 (1967), 373-383.

46. H. Moskowirz, Effects of problem representation and feedback on rational behavior in Allais- and Morlat-type problems, Decision Sci. 5 (1974), 225-242.

47. J. Mossin, A note on uncertainty and preferences in a temporal context, Amer. Econ. Rev. 59 (1969), 172-174.

48. D. C. NaChmaN, Risk aversion, impatience, and optimal timing decisions, J. Econ. Theory 11 (1975), 196-246.

49. E. H. Neave, Multiperiod consumption-investment decisions and risk preference, $J$. Econ. Theory 3 (1971), 40-53.

50. P. Nogee And B. Lieberman, The auction value of certain risky situations, J. Psychol. $49(1960), 167-179$.

51. E. S. Phelps, The accumulation of risky capital: A sequential utility analysis, Econometrica 30 (1962), 729-743.

52. J. W. Pratt, Risk aversion in the small and in the large, Econometrica 32 (1964), $122-136$.

53. M. G. Preston and P. Baratta, An experimental study of the auction-value of an uncertain outcome, Amer. J. Psychol. 61 (1948), 183-193.

54. H. RAIFFA, "Decision Analysis: Introductory Lectures on Choices under Uncertainty." Addison-Wesley, Reading, 1968.

55. R. T. Rockafellar, "Convex Analysis," Princeton Univ. Press, Princeton, N. J. 1970.

56. S. A. Ross, Some stronger measures of risk aversion in the small and the large with applications, Econometrica 49 (1981), 621-638.

57. M. Rossman and L. Selden, "Nutes on Indirect Cardinal Utilily and Intertemporal Choice under Uncertainty," Columbia University Dept. of Economics Discussion Paper No. $48,1979$.

58. M. Rothschild AND J. E. Stiglitz, Increasing risk. I. A definition, J. Econ. Theory 2 (1970), 225-243.

59. M. Rothschild and J. E. Stiglitz, Increasing risk. II. Its economic consequences, $J$. Econ. Theory 3 (1971), 66-84.

60. W. Rudin, "Functional Analysis," McGraw-Hill, New York, 1973.

61. R. R. Russell AND M. Wilkinson, "Microeconomics: A Synthesis of Modern and Neoclassical Theory," Wiley, New York. 1979.

62. P. A. Samuelson, Utility, preference, and probability, in "Collected Scientific Papers of Paul A. Samuelson, Volume 1" (J. E. Stiglitz, Ed.), Chap. 13, MIT Press, Cambridge, Mass., 1966.

63. A. SANDmo, Portfolio choice in a theory of saving, Swedish J, Econ. 70 (1968), 106-122.

64. A. SANDmo, Capital risk, consumption, and portfolio choice, Econometrica 37 (1969), 586-599.

65. A. SANDmo, The effect of uncertainty on saving decisions, Rev. Econ. Stud. 37 (1970), 353-360.

66. L. SELDEN, A new representation of preferences over "certain $\times$ uncertain" consumption pairs: The "ordinal certainty equivalent" hypothesis, Econometrica 46 (1978), 1045-1060. 
67. P. Slovic ANd A. TVERSKy, Who accepts Savage's axiom?, Behav. Sci. 19 (1974), 368-373.

68. M. Spence AND R. ZeCKhauser, The effect of the timing of consumption decisions and the resolution of lotteries on the choice of lotteries, Econometrica 40 (1972), 401-403.

69. R. C. Sprowls, Psychological-mathematical probability in relationships of lottery gambles, Amer. J. Psychol. 66 (1953), 126-130.

70. L. Tesfatsion, Stochastic dominance and the maximization of expected utility, Rev. Econ. Stud. 43 (1976), 301-315.

71. A. TVERSky, Utility theory and additivity analysis of risky choices, J. Exp. Psychol. 75 (1967), 27-36.

72. A. Tversky, Additivity, utility, and subjective probability, J. Math. Psychol. 4 (1967), $175-201$.

73. A. TVERSKy, A critique of expected utility theory: Descriptive and normative considerations, Erkenntnis 9 (1975), 163-173.

74. H. Varian, "Microeconomic Analysis," Second Edition, Norton, New York, 1984.

75. R. Weber, The Allais Paradox, Dutch auctions, and alpha-utility theory, unpublished manuscript, J. L. Kellogg Graduate School of Management, Northwestern University, 1982.

76. G. A. Whitmore, Third-degree stochastic dominance, Amer. Econ. Rev. 60 (1970), 457-459.

77. M. E. YAARI, Some remarks on measures of risk aversion and on their uses, J. Econ. Theory 1 (1969), 315-329. 